\title{
Measuring the impact of olive pomace enriched biscuits on the gut microbiota and its metabolic activity in mildly hypercholesterolaemic subjects
}

\author{
Lorenza Conterno $^{1} \cdot$ Francesca Martinelli $^{1} \cdot$ Matteo Tamburini $^{1} \cdot$ Francesca Fava $^{3}$ - Andrea Mancini $^{3}$.

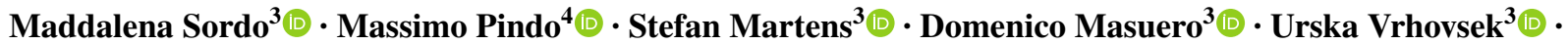 \\ Claudia Dal Lago $^{2} \cdot$ Gabriele Ferrario $^{2} \cdot$ Mario Morandini $^{1} \cdot$ Kieran Tuohy $^{3}$ (i)
}

Received: 31 May 2017 / Accepted: 24 October 2017 / Published online: 9 November 2017

(C) The Author(s) 2017. This article is an open access publication

\begin{abstract}
Purpose Olive pomace is a major waste product of olive oil production but remains rich in polyphenols and fibres. We measured the potential of an olive pomace-enriched biscuit formulation delivering $17.1 \pm 4.01 \mathrm{mg} / 100 \mathrm{~g}$ of hydroxytyrosol and its derivatives, to modulate the composition and metabolic activity of the human gut microbiota.

Methods In a double-blind, controlled parallel dietary intervention 62 otherwise healthy hypercholesterolemic (total plasma cholesterol $180-240 \mathrm{mg} / \mathrm{dl}$ ) subjects were randomly assigned to eat $90 \mathrm{~g}$ of olive pomace-enriched biscuit (olive-enriched product, OEP) or an isoenergetic control (CTRL) for 8 weeks. Fasted blood samples, 24-h urine and faecal samples were collected before and after dietary intervention for measurement of microbiota, metabolites and clinical parameters.

Results Consumption of OEP biscuits did not impact on the diversity of the faecal microbiota and there was no statistically significant effect on CVD markers. A trend towards reduced oxidized LDL cholesterol following OEP ingestion was observed. At the genus level lactobacilli and Ruminococcus were reduced in OEP compared to CTRL biscuits.
\end{abstract}

Kieran Tuohy

kieran.tuohy@fmach.it

1 OlioCRU s.r.l. Research and Development Group, Via Aldo Moro 1, 38062 Arco, TN, Italy

2 Casa di Cura Eremo di Arco s.r.1., Via XXI Aprile 1, 38062 Arco, TN, Italy

3 Department of Food Quality and Nutrition, Research and Innovation Centre-Fondazione Edmund Mach, San Michele all'Adige, Italy

4 Genomics and Advanced Biology Unit, Research and Innovation Centre-Fondazione Edmund Mach, San Michele all'Adige, Italy
A trend towards increased bifidobacteria abundance was observed after OEP ingestion in 16S rRNA profiles, by fluorescent in situ hybridization and by qPCR. Targeted LC-MS revealed significant increases phenolic acid concentrations in 24-h urine following OEP ingestion and 3,4-dihydroxyphenylacetic acid (DOPAC) and homovanillic acid, derivatives of hydroxytyrosol, were elevated in blood. A sex effect was apparent in urine small phenolic acid concentrations, and this sex effect was mirrored by statistically significant differences in relative abundances of faecal bacteria between men and women.

Conclusion Ingestion of OEP biscuits led to a significant increase in the metabolic output of the gut microbiota with an apparent sex effect possibly linked to differences in microbiota makeup. Increased levels of homovanillic acid and DOPAC, thought to be involved in reducing oxidative LDL cholesterol, were observed upon OEP ingestion. However, OEP did not induce statistically significant changes in either ox-LDL or urinary isoprostane in this study.

Keywords Olive product $\cdot$ Prebiotic $\cdot$ Polyphenols · Metabolomic · Tyrosol glucoside · Tyrosol group

\section{Introduction}

Olives and olive oil are important and characteristic components of the Mediterranean diet, a dietary pattern shown to improve on both physical and mental quality of life, and reduce the risk of chronic diet-associated disease, especially cardiovascular disease (CVD) [1]. Indeed, extra-virgin olive oil, as part of a Mediterranean style diet significantly reduced both the incidence of composite CVD end points and total mortality in the PREDIMED study [2]. Polyphenols, complex aromatic plant secondary metabolites, are 
independently linked to these health effects [1,3]. Olives and various olive oils and extracts have been shown to mediate different health effects in humans, many associated with CVD risk. Olive extracts have been reported to lower systolic blood pressure (SBP) and diastolic blood pressure (DBP) from baseline in both hypertensive and pre-hypertensive individuals [4-7] and to improve plasma lipid profiles in both normo-lipidaemic and hypercholesterolaemic subjects $[4,6,8-10]$. Olive extracts have also been found to induce acute reductions in arterial stiffness [11], which agrees with data suggesting that olive oil and olive extract significantly improve vascular function [12, 13] and blood pressure [14]. Currently, these improvements are thought to be associated with polyphenol-rich olive oil fractions rather than other bioactives which may be present $[15,16]$. In contrast, other studies have failed to demonstrate significant modulation of CVD biomarkers upon olive extract ingestion. For example, olive leaf extracts did not appear to improve plasma lipids [7, 17], ambulatory blood pressure, cytokines and/or carotid intima-media thickness [17] in different studies. However, the European Food Safety Authority (EFSA) has recognized a specific health claim for the polyphenol extract of olive for protection of LDL cholesterol particles against oxidative damage - although they also noted that there is a lack of evidence for other health claims including maintenance of normal blood pressure and HDL cholesterol levels, reduced inflammation and improved gastrointestinal function [18]. EFSA considers that the claim that "consumption of olive oil polyphenols contributes to the protection of blood lipids from oxidative damage" reflects the scientific evidence, and that a dose of $5 \mathrm{mg}$ of hydroxytyrosol and its derivatives (e.g. oleuropein complex and tyrosol) in olive oil should be consumed daily for food products to bear the health claim. In olive, the majority of polyphenols present belong to the tyrosol group [hydroxytyrosol (HT), tyrosol (TYR) and conjugated forms like oleuropein]. These conjugated forms are extensively hydrolyzed in the stomach [19] to HT and TRY, which are either absorbed in the small intestine and undergo extensive phase I and II biotransformation or reach the colon where they undergo biotransformation by the resident microbiota $[19,20]$. The most common derivatives are small phenolic acids like homovanillic acid (HVA), dihydroxyphenylacetic acids (DHPAA), hydroxyphenylacetic acid (HPAA), protocatechuic acid and benzoic acids for example. The impact of olives or their constituent parts on the composition and metabolic activity of the human intestinal microbiota is, however, poorly understood. One recent study has shown that thyme phenolic compounds at different doses in olive oil can induce a small increase in bifidobacteria using the quantitative culture-independent method fluorescent in situ hybridization (FISH), with the suggestion that this change in microbiota could be related to improved LDL cholesterol oxidative status [21]. Similarly, a sex effect in terms of metabolism of HT and related compounds has been observed in rats with the suggestion that differential excretion of HT derivatives between male and female animals might be due to sex-linked differences in enterohepatic circulation [22]. However, no data are reported for differences in metabolism of these compounds between men and women or indeed, whether such differences if they do exist, could be due to sex-specific differences in gut microbiota. To date, no studies have reported whether olives or olive pomace can impact on the relative abundances of the human gut microbiota, on the diversity of the gut microbiota and only a few studies have specifically addressed the metabolic end products produced by combined host-microbiota cometabolism of olive polyphenols [20,21].

Olives contain many potential biologically active compounds such as polyphenols, dietary fibre (including pectin), oleic acid, linoleic acid and other beneficial fats, tocopherols, phytosterols and squalene. Olive oil is extracted from the fruit of Olea europaea, leaving waste in the form of olive water and solid olive pomace. The olive pomace and wastewater produced from oil extraction processes contain macromolecules such as polysaccharides, lipids, proteins and polyphenolic compounds (mainly of the tyrosol group) which can range from 1 to $8 \mathrm{~g} / 1$ [23] in wastewater and 2.9 to $3.7 \mathrm{mg} / \mathrm{l}$ in olive pomace [24]. The annual worldwide production of olive oil is about 2 million metric tons reported for 2015/16 (average: COI, 2017). For each ton of olive oil the waste produced is dependent on the fruit quality, ripeness and extraction technology and typically ranges from 2.75 to 4 tons of olive pomace and $1-8 \mathrm{~m}^{3}$ of wastewater [25-27]. In addition these olive mill wastes are produced in significantly large quantities during the short olive production season and because the waste cannot be disposed of through ordinary waste treatment systems, disposal of wastewater and olive pomace is a major environmental problem and cost to the industry. Given the push towards a green economy, there is a stimulus for the oil industry to move towards a circular economic model, reducing waste production or adding value to waste streams, developing added value secondary product lines. To this end, we have developed an olive pomace extract as a functional food ingredient. This olive pomace, which delivers $17.1 \pm 4.01 \mathrm{mg} / 100 \mathrm{~g} \mathrm{HT}$ and its derivatives, is also rich in fibres and our preliminary in vitro data showed it was fermentable and leads to increased numbers of bifidobacteria in $\mathrm{pH}$-controlled faecal batch cultures (data not shown). For this current study, we have incorporated this olive pomace into a biscuit formulation. For flavour, texture and constitutional reasons, other ingredients were also necessary including extra virgin olive oil and flours from chestnut, pea and buckwheat, but the olive pomace remained the dominant ingredient and the dominant source of polyphenols (>90\% of polyphenols present in the final biscuit). Here we report the effect of this olive pomace-enriched biscuit on 
the human gut microbiota, their metabolic output and on various biomarkers of CVD and inflammation. The study was conducted jointly by researchers at Fondazione Edmund Mach (San Michele all'Adige, Italy), OlioCru s.r.l (Arco, Italy) and the study centre was the Casa di Cura Eremo di Arco s.r.l. (Italy), a private specialist clinic specialized in treating CVD.

\section{Materials and methods}

\section{Study design}

The study was a double-blind, randomized, controlled, parallel trial (Italy protocol Prebioil2 number: 6/2015, Clinicaltrials.gov ID: NCT02664428, see Fig. 1 for the study design). This study was conducted according to the guidelines laid down in the Declaration of Helsinki, and all procedures involving human subjects were approved by the Ethic Committee for Clinical Trials of the Trento Azienda Provinciale Servizi Sanitari (APSS). The clinical trial was carried out at the Casa di Cura Eremo di Arco (Arco, TN, Italy) between

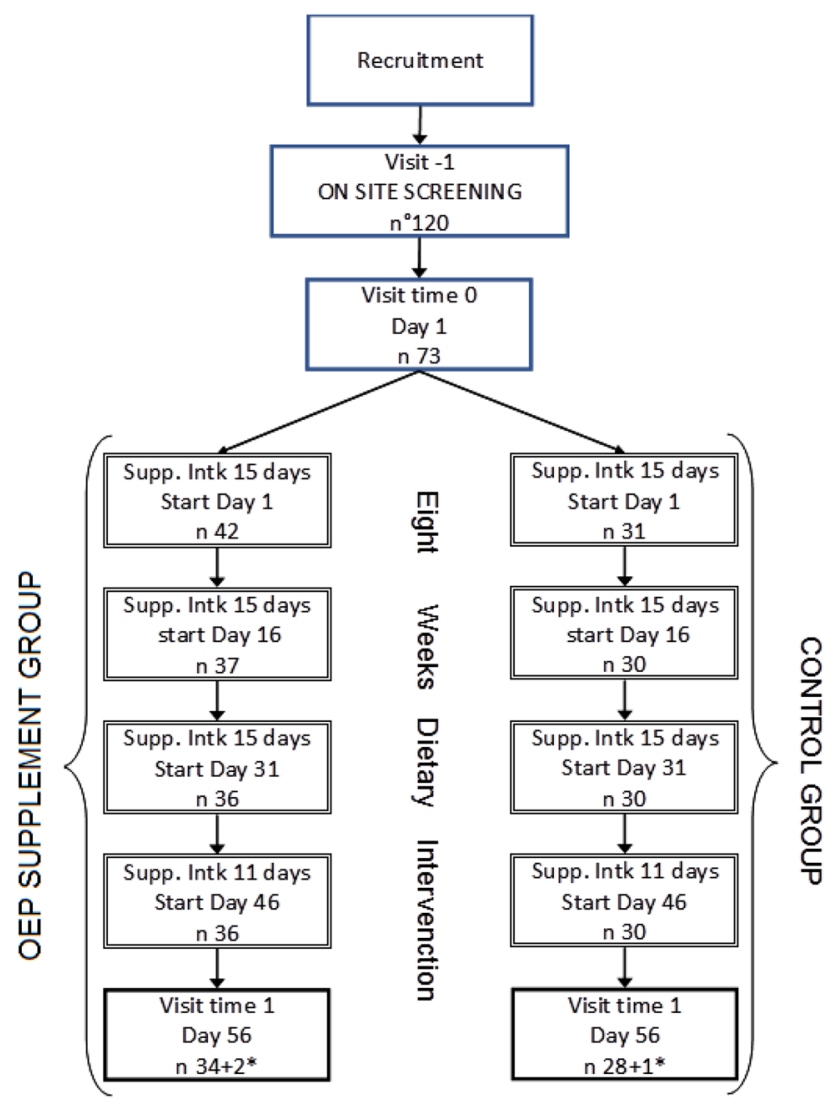

Fig. 1 Schematic representation of study design. *At the end of the study because of antibiotic usage or non-compliance for consumption of the product, two people were excluded from the olive-enriched product (OEP) group and one from the control group
November 2015 and June 2016. The primary outcome measure was the faecal microbiota analysis by Illumina sequencing and fluorescence in situ hybridization (FISH) carried out with probe for Bifidobacterium spp., Lactobacillus spp. and Ruminococcus spp. The secondary outcome measures were related to cholesterol analysis in plasma [total, LDL, HDL cholesterol, oxidized LDL, triglycerides, apolipoproteins A-I and B (APO A and APO B)] together with the analysis of the variation of polyphenols and their metabolites in plasma and urine. Additional measures were the analyses of the anthropometric indices, the fasting plasma insulin, glucose and C-reactive protein (CRP) and the analysis of isoprostane $\mathrm{F} 2$ in urine. This study was powered for changes in blood LDL cholesterol and changes in faecal bifidobacteria. Since previous studies have shown that fewer individuals are required for measuring changes in faecal bifidobacteria and because of its clinical significance, the sample size calculation was performed only for changes in LDL cholesterol levels. Based on measures from a previous parallel trial design using similar products [28], we assessed as clinical significant end point the average LDL decrease of $15.44 \mathrm{mg} / \mathrm{dl}$ with a standard deviation of $14.28 \mathrm{mg} / \mathrm{dl}$. According to Snedecor and Cochran equation with significance value $\alpha$ equal to 0.05 and a $90 \%$ power $(1-\beta)$ [29], the minimum number of subjects to enrol was 28 . Taking into account possible dropouts, a total of 73 subjects were enrolled and randomly assigned to the dietary intervention with the product under investigation (OEP) or to the control dietary intervention.

\section{Intervention foods}

The study product "PreBiÒ ${ }^{\circledR ”, ~}$, herein called olive pomaceenriched product (OEP), was a bakery product comprising dehydrated food-grade olive powder, together with chestnut, peas, buckweat flour, extra virgin olive oil (EVOO), salt and sugar according to the OlioCRU proprietary recipe, and the OlioCRU pending Patent Process. The product was prepared at a local bakery. The product contained about $411 \pm 25 \mathrm{mg} / 100 \mathrm{~g}$ of total biophenols measured according to the COI method of which $17.1 \pm 4.01 \mathrm{mg} / 100 \mathrm{~g}$ belong to the tyrosol group polyphenols and were measured according to Gasperotti et al. [30].

The product corresponded to the energy intake of $434 \mathrm{Kcal} / 100 \mathrm{~g}$ (average of three replicates).

Subjects were provided with single $90 \mathrm{~g}$ daily doses and instructed to consume one each day of the 8 weeks of intervention. The control product comprised wheat flour, sugar, salt and low-polyphenol EVOO, food colourings in safety-approved quantities to match the test product OEP as closely as possible in appearance, taste, texture. The product contains less than $1 \mathrm{mg} / 100 \mathrm{~g}$ of total biophenols measured according to the IOC official method (IOC 2009) of which $0.7 \pm 0.5 \mathrm{mg} / 100 \mathrm{~g}$ belong to the tyrosol group. The product 
corresponds to an energy intake of $419 \mathrm{Kcal} / 100 \mathrm{~g}$ (average of three replicates). Table 1 shows the gross nutritional composition of the OEP and control (CTRL) biscuits.

\section{Recruiting}

Healthy volunteer were recruited through advertisement in the geographical area of Arco (TN) in the northeast of Italy. Advertisement was carried out via flyer, posters and e-mails. Individuals who answered the call were asked their weight and height and those who corresponded to BMI in the range established by the inclusion criteria were given an appointment for the general health assessment to determine conformity with other inclusion/exclusion criteria. At the clinic a health and lifestyle questionnaire was completed, eco-cardiogram and physical examinations were performed together with collection of urine and fasted blood samples. The inclusion criteria were non-smoking status, age between 30 and 65 years, BMI between 20 and $29.9 \mathrm{~kg} / \mathrm{m}^{2}$, plasmatic total cholesterol between 180 and $240 \mathrm{mg} / \mathrm{dl}$, being free from chronic disease, including cardiovascular disease, diabetes, cancer, inflammatory or digestive disorders. Pregnancy or breastfeeding, and individuals consuming more than $21 \mathrm{U} /$ week of alcohol were excluded. Subjects were excluded if taking statins or other medication or dietary supplements that may affect lipids. Uncontrolled hypertension was an exclusion criteria, and to be included the subjects were either not hypertensive or under hypertensive medication and presenting with average SBP below $121 \mathrm{mmHg}$ and average DBP $90 \mathrm{mmHg}$. Subjects with food allergies or intolerances were also excluded.

\section{Randomisation and blinding}

Treatment allocation was done using a random block design. A six-digit code was assigned to each recruited individual. The products was randomly assigned to the subject code by an external individual by picking codes form a bag in a blind manner but matching groups by age and sex. Each daily dose was prepared in a food-grade sealed box enveloped in dark green paper sachet. The treatment codes were kept offsite and not released until statistical analysis was complete. Therefore, allocation concealment was achieved and both researchers and subjects were blinded to which product was being consumed at which time.

\section{Screening}

Clinical visits took place at the beginning of week 1, and at the end of week 8 at the Casa di Cura Eremo di Arco s.r.l., Via XXI Aprile 1, 38062 Arco (TN) Italy. Subjects arrived for screening fasted and measurements of height and weight were taken in a Kern scale (Kern \& Sohn, Balingen,
Germany) with stand and height rod (Mod MPB300K100P) to calculate BMI. Blood pressure was measured after 5-min rest, seated and with the subject's dominant arm resting on a table, using an Omron digital blood pressure equipment (HEM-705 CP).

Three readings were taken $60 \mathrm{~s}$ apart and averaged. Subjects were not permitted to talk during measurements. Blood pressure was measured at the screening visit (T-1) and later at the beginning (T0) and at the end of the treatment (T1). Blood samples obtained via single venepuncture were collected into heparin and EDTA vacutainers (BD) and used for the analysis established for health. A total of 73 suitable subjects were identified and accepted onto the trial.

Each subject was informed about the study aims and procedure to allow them to sign and informed consent. Eligible participants were asked to provide written informed consent to take part in the study.

\section{Compliance measures}

Subjects were asked to return all remaining full or empty daily packages of test product after 8 week intervention. Remaining material were weighed and recorded. Subjects were asked to complete weekly online questionnaires and supplied with daily tick sheets.

\section{Faecal sample collection}

Volunteers were provided with a sealable pot, and sterile bag to collect the stool sample, each of them were instructed to collect the stool sample in the sterile bag, put it into the pot and, prior to sealing the pot, to add an atmosphere generation system AnaeroGen Compact (Thermo Scinetific). This ensured an anaerobic environment during sample transport. Samples were collected and treated for further analysis or stored at $-80^{\circ} \mathrm{C}$ within $24 \mathrm{~h}$.

\section{Urine sample collection}

Volunteers were supplied 3-1 sterile containers to collect the 24-h urine samples. Each container was added with $15 \mathrm{ml}$ of $3 \mathrm{M}$ hydrochloridric acids as a preservative. After collection, the total urine volume was measured and the samples for further analysis were prepared and stored at $-80^{\circ} \mathrm{C}$.

\section{Biochemical measures}

Blood collected in EDTA and heparin vacutainers was centrifuged at $1550 \times g$ for $15 \mathrm{~min}$ to separate plasma. Plasma was immediately analysed or stored in low-binding Eppendorf tubes (Axygen, Tewksbury MA, USA) at $-80^{\circ} \mathrm{C}$ until analysis. Total cholesterol (TC), HDL cholesterol (HDL), LDL cholesterol (LDL), apolipoprotein A1 (Apo A1) and B 
Table 1 Food supplements' nutritional value (g/100 g, mean \pm standard deviation) (a) and polyphenol composition $(\mu \mathrm{g} / \mathrm{g})(\mathbf{b})$

\begin{tabular}{|c|c|c|c|c|}
\hline & \multicolumn{2}{|c|}{ OPE (g/100 g) } & & $\mathrm{Ctrl}(\mathrm{g} / 100 \mathrm{~g})$ \\
\hline \multicolumn{5}{|l|}{ A } \\
\hline Carbohydrates & \multicolumn{2}{|c|}{$49.2( \pm 1.04)$} & \multicolumn{2}{|r|}{$70.9( \pm 2.19)$} \\
\hline Lipids & \multicolumn{2}{|c|}{$18.5( \pm 0.40)$} & \multicolumn{2}{|r|}{$8.1( \pm 2.40)$} \\
\hline Proteins & \multicolumn{2}{|c|}{$16.1( \pm 4.39)$} & \multicolumn{2}{|r|}{$10.9( \pm 0.92)$} \\
\hline Dietary fibre & \multicolumn{2}{|c|}{$13.3( \pm 0.17)$} & \multicolumn{2}{|r|}{$3.4( \pm 0.35)$} \\
\hline Salt & \multicolumn{2}{|c|}{$0.7( \pm 0.2)$} & \multicolumn{2}{|r|}{$0.7( \pm 0.2)$} \\
\hline \multirow[t]{2}{*}{ Compound } & \multicolumn{2}{|l|}{ OEP } & \multicolumn{2}{|l|}{ Ctrl } \\
\hline & Mean & SD & Mean & SD \\
\hline \multicolumn{5}{|l|}{ B } \\
\hline Cinnamic acid & 0.39 & 0.04 & $<0.005$ & - \\
\hline Vanillin & 0.26 & 0.02 & $<0.01$ & - \\
\hline Esculin & 0.14 & 0.01 & $<0.005$ & - \\
\hline Neochlorogenic acid & 0.40 & 0.18 & $<0.01$ & - \\
\hline Chlorogenic acid & 55.08 & 4.24 & $<0.025$ & - \\
\hline Fertaric acid & 0.09 & 0.02 & $<0.01$ & - \\
\hline$t$-Coutaric acid & 0.68 & 0.13 & $<0.01$ & - \\
\hline Apigenin & 3.30 & 0.13 & $<0.0025$ & - \\
\hline Luteolin & 10.14 & 3.40 & 1.19 & 1.26 \\
\hline Luteolin-7-O-Glc & 8.95 & 0.88 & $<0.0025$ & - \\
\hline Apigenin-7-Glc & 1.23 & 0.07 & $<0.0025$ & - \\
\hline Naringenin & 0.17 & 0.03 & $<0.0025$ & - \\
\hline Epicatechin & 0.48 & 0.36 & $<0.1$ & - \\
\hline Kaempferol & 3.44 & 0.52 & $<0.0025$ & - \\
\hline Quercetin & 2.57 & 0.26 & $<0.0025$ & - \\
\hline Quercetin-3-Rha & 1.52 & 0.11 & $<0.005$ & - \\
\hline Kaempferol-3-Glc & 2.25 & 0.39 & $<0.0025$ & - \\
\hline $\begin{array}{l}\text { Syringetin-3-Glc + syringetin-3-Gal (as syr- } \\
\text { 3-glc) }\end{array}$ & 0.14 & 0.02 & $<0.0025$ & - \\
\hline Kaempferol-3-rutinoside & 0.07 & 0.02 & $<0.0025$ & - \\
\hline Arbutin & 0.21 & 0.05 & 0.31 & 0.11 \\
\hline 4-Hydroxybenzoic acid & 1.02 & 0.33 & 0.81 & 0.12 \\
\hline$p$-Coumaric acid & 1.01 & 0.38 & 0.71 & 0.09 \\
\hline Vanillic acid & 2.98 & 0.43 & 1.37 & 0.18 \\
\hline Caffeic acid & 1.19 & 0.11 & $<0.025$ & - \\
\hline Isoferulic acid & 0.74 & 0.03 & 1.04 & 0.18 \\
\hline$t$-Ferulic acid & 21.21 & 0.03 & 33.65 & 4.75 \\
\hline Ellagic acid & 6.40 & 1.26 & $<0.025$ & - \\
\hline Pyrocatechol & 7.20 & 0.60 & 9.09 & 2.36 \\
\hline Protocatechuic acid & 4.69 & 0.65 & $<0.025$ & - \\
\hline Scopoletin & 0.26 & 0.08 & $<0.0025$ & - \\
\hline Cryptochlorogenic acid & 3.65 & 0.64 & $<0.025$ & - \\
\hline $\begin{array}{l}\text { Quercetin-3-Glc + quercetin-3-gal(as que-3- } \\
\text { glc) }\end{array}$ & 0.60 & 0.37 & $<0.005$ & - \\
\hline Rutin & 2.32 & 0.42 & $<0.0025$ & - \\
\hline Hydroxytyrosol & 8.11 & 4.41 & 0.79 & 0.32 \\
\hline Chrysoeriol & 0.13 & 0.02 & 0.07 & 0.02 \\
\hline Salidroside & 121.19 & 34.59 & $<0.25$ & - \\
\hline Pinoresinol & 1.11 & 0.15 & $<0.0025$ & - \\
\hline Oleuropein & 5.72 & 0.78 & $<0.005$ & - \\
\hline
\end{tabular}


Table 1 (continued)

\begin{tabular}{lcccc}
\hline Compound & \multicolumn{2}{l}{ OEP } & \multicolumn{2}{c}{ Ctrl } \\
\cline { 2 - 4 } \cline { 3 - 5 } & Mean & SD & Mean & 0.06 \\
\hline Diosmetin & 0.13 & 0.03 & 0.01 \\
Tyrosol & 8.86 & 0.86 & 6.31 & 0.72 \\
\hline
\end{tabular}

Values below the limit of quantification are not shown

(Apo B) triglycerides (TG), CRP, glucose (GLU) and insulin (Ins) were measured at Clinic Casa di Cura Eremo Laboratory (Arco, TN, Italy), using ILab 650 chemistry analyser (Instrumentation Laboratories UK Ltd, Warrington, United Kingdom) for all measures except insulin which was measured using a Roche COBAS 6000.

Oxidized LDL was measured in duplicate via an ELISA kit (Mercodia, Sweden).

F2 isoprostane urinary total (conjugated and non-conjugated) were measured using the enzyme immunoassay based kit Urinary Isoprostane Elisa Kit (Oxford Biomedical Research, USA).

\section{Metagenomic analysis}

A whole fresh stool sample was collected at T0 and T1. The stool was stored at $-80{ }^{\circ} \mathrm{C}$. DNA was extracted from $100 \mathrm{mg}$ stool aliquots using Power faecal DNA extraction kit (MOBio), following the manufacturer's instructions. Metagenomic sequencing was performed to evaluate microbiota diversity and genus-level abundances. Using the specific bacterial primer set 341F (5' CCTACGGGNGGCWGCAG 3') and 806R (5'GACTACNVGGGTWTCTAATCC $3^{\prime}$ ) with overhang Illumina adapters, total genomic DNA was subjected to PCR amplification by targeting a 460-bp fragment of the 16S rRNA variable region V3-V4. PCR amplification of each sample was carried out using $25-\mu$ l reactions with $1 \mu \mathrm{M}$ of each primer. Specifically $12.5 \mu \mathrm{l}$ of $2 \times$ KAPA HiFi HotStart ReadyMix, $5 \mu$ l forward primer, $5 \mu$ reverse primer were used in combination with $2.5 \mu \mathrm{l}$ of template DNA (5 ng/ $\mu \mathrm{l})$. All PCR amplifications were carried out using a GeneAmp PCR System 9700 (Thermo Fisher Scientific) and the following steps-melting step; $94^{\circ} \mathrm{C}$ for 5 min (one cycle), annealing step; $95^{\circ} \mathrm{C}$ for $30 \mathrm{~s}, 55^{\circ} \mathrm{C}$ for $30 \mathrm{~s}, 72{ }^{\circ} \mathrm{C}$ for $30 \mathrm{~s}$ ( 30 cycles), extension step; $72{ }^{\circ} \mathrm{C}$ for 5 min ( 1 cycle). The PCR products were checked on $1.5 \%$ agarose gel and cleaned from free primers and primer dimer using the Agencourt AMPure XP system (Beckman Coulter, Brea, CA, USA) following the manufacturer's instructions. Subsequently dual indices and Illumina sequencing adapters Nextera XT Index Primer (Illumina) were attached by sevencycle PCR (16S Metagenomic Sequencing Library Preparation, Illumina). The final libraries, after purification by the Agencourt AMPure XP system (Beckman), were analysed on a Typestation 2200 platform (Agilent Technologies, Santa Clara, CA, USA) and quantified using the Quant-IT PicoGreen dsDNA assay kit (Thermo Fisher Scientific) by the Synergy 2 microplate reader (Biotek). Finally all the libraries were pooled in an equimolar way in a final amplicon library and analysed on a Typestation 2200 platform (Agilent Technologies, Santa Clara, CA, USA). Bar-coded library were sequenced on an Illumina ${ }^{\circledR}$ MiSeq (PE300) platform (MiSeq Control Software 2.0.5 and Real-Time Analysis software 1.16.18). Differences in relative abundance after intervention (V2-V1) with treatment $\mathrm{W}$ or $\mathrm{P}$ were analysed using non-parametric $t$ test (Mann-Whitney $U$ test).

\section{Quantitative microbial molecular techniques}

\section{Flow cytometry (FCM) fluorescent in situ hybridization (FISH)}

1:10 dilution (wt:vol) of the faecal sample was prepared by weighting out 2-3 g of faecal sample and diluting it with $1 \mathrm{M}$ PBS on the scale (e.g. $3 \mathrm{~g}$ of sample $+27 \mathrm{~g}$ of PBS, considering that $1 \mathrm{ml}$ of $1 \mathrm{M}$ PBS weights $1 \mathrm{~g}$ ). The diluted sample was homogenized using a Stomacher 400 (Seward) at the speed of $230 \mathrm{rpm}$ for $2 \mathrm{~min}$ or until it appeared homogeneous. Ten millilitre of the suspension was transferred into a $15-\mathrm{ml}$ falcon tube containing glass beads; the tube was mixed by vortexing for about $30 \mathrm{~s}$, then centrifuged at $1100 \mathrm{rpm}$ for $2 \mathrm{~min}$ to pellet fibrous particles. Then $375 \mu \mathrm{l}$ of the suspension were transferred into a 1.5-ml Eppendorf tube containing $1125 \mu \mathrm{l}$ of $4 \%$ paraformaldehyde (PFA). The suspension was fixed in 4\% PFA for $4-24 \mathrm{~h}$ at $4{ }^{\circ} \mathrm{C}$. After fixation the tubes were centrifuged at $13,000 \mathrm{rpm}$ for $5 \mathrm{~min}$ and the pellet were resuspended in $1 \mathrm{ml}$ of filtersterilized 1M PBS. This washing procedure was repeated twice, then the tubes were centrifuged again at 13,000 rpm for $5 \mathrm{~min}$, supernatant was carefully removed and the pellet was finally resuspended in $150 \mu \mathrm{l}$ of filter-sterilized $1 \mathrm{M}$ PBS. One hundred and fifty (150) microlitre of absolute ethanol was added and the samples were mixed by inversion and immediately stored at $-20{ }^{\circ} \mathrm{C}$. For FCM-FISH analysis, $10 \mu \mathrm{l}$ of the fixed faecal sample was resuspended in $190 \mu \mathrm{l}$ of PBS $1 \mathrm{X}$ sterile. Every step was done in 96-well plates. After resuspending, the sample was centrifuged at 
$4000 \mathrm{rpm}$ for $15 \mathrm{~min}$. The sample was resuspended in $200 \mu \mathrm{l}$ of Tris-EDTA buffer and then centrifuged another time at $4000 \mathrm{rpm}$ for $15 \mathrm{~min}$. For hybridisation with the probes specific for Lactobacillus/Enterococcus spp. [31], Bifidobacterium spp. [32] and Ruminococcus spp. [33] that needed lysozyme treatment to render the cell wall more permeable to the probes, we resuspended the sample in $200 \mu \mathrm{l}$ of Tris-EDTA containing $1 \mathrm{mg} / \mathrm{ml}$ of lysozyme and incubated for $10 \mathrm{~min}$ at room temperature. After that period, sample was washed with centrifugation and resuspended in $50 \mu 1$ of hybridization buffer [0.9 M NaCl, $20 \mathrm{mM}$ Tris- $\mathrm{HCl}$ (pH $7.5), 0.1 \%$ [wt/vol] sodium dodecyl sulphate (SDS)] with $5 \mu \mathrm{l}$ of $50 \mathrm{ng} / \mu \mathrm{l}$ fluorescently Cy5-labelled oligonucleotide probe and incubated at the appropriate hybridization temperature (Table 2). Washing was repeated and the sample was resuspended in $200 \mu \mathrm{l}$ of hybridization buffer without SDS and incubated at the appropriate wash temperature (Table 2). After washing, sample was resuspended in SYBR Green, used to enumerate the total cells. SYBR Green binds to DNA and the resulting complex absorbs blue light $\left(\lambda_{\max }=497 \mathrm{~nm}\right)$ and emits green light $\left(\lambda_{\max }=520 \mathrm{~nm}\right)$. A blank sample (without the fluorescently Cy5-labelled oligonucleotide probe and without the SYBR Green) was prepared and for every sample, following the same steps as per the hybridized sample, to set the threshold of the gates of the flow cytometer that permits the revelation of the microbial species and exclude the false positives due to the potential autofluorescence of the sample. FCM was performed using Guava easyCyte ${ }^{\mathrm{TM}}$ Single Sample Flow Cytometer (Millipore) with a single blue (488 nm), dual blue and red (642 nm), or triple blue, red, and violet $(405 \mathrm{~nm})$ excitation lasers that provided 12 simultaneous detection parameters, including 10 fluorescent colours plus forward and side scatter for size and granularity determination. The FCM parameters were adjusted to give particle counts of 1000 events in total. Data were analysed by the InCyte software, version 4.1.1. To avoid loss of the signal intensity of hybridized cells, the samples were kept in the dark until the FCM analysis. Results were expressed as the percentage of cells hybridized with the group-specific-Cy5 probe calculated on the total bacteria, counted after SYBR Green staining. Also absolute numbers were obtained.

\section{Quantitative PCR}

DNA extraction was performed using the FastDNA ${ }^{\mathrm{TM}}$ SPIN Kit for Feces (MP Biomedicals). Amplifications were performed with sets of primers specific for Bifidobacterium
Table 2 Anthropometric and clinical parameters, mean ( \pm standard deviation), before (T0) and after (T1) dietary intervention with olive-enriched product (OEP) or control (Ctrl)

\begin{tabular}{|c|c|c|c|c|c|}
\hline & \multicolumn{2}{|c|}{ Dietary supplement } & \multicolumn{2}{|c|}{ Dietary supplement } & \multirow{3}{*}{$\begin{array}{l}\text { ANOVA } \\
p \text { value }\end{array}$} \\
\hline & \multicolumn{2}{|l|}{ OEP } & \multicolumn{2}{|l|}{ Ctrl } & \\
\hline & T0 & $\mathrm{T} 1$ & T0 & $\mathrm{T} 1$ & \\
\hline SYS (mmHg) & $120( \pm 11.4)$ & $122( \pm 12.4)$ & $122( \pm 15.0)$ & $121( \pm 17.2)$ & 0.416 \\
\hline DIA (mmHg) & $78( \pm 7.4)$ & $78( \pm 7.7)$ & $78( \pm 9.7)$ & $77( \pm 9.8)$ & 0.586 \\
\hline $\mathrm{WM}(\mathrm{cm})$ & $84( \pm 11.9)$ & $84( \pm 11)$. & $83( \pm 11.7)$ & $82( \pm 10.9)$ & 0.931 \\
\hline $\mathrm{HM}(\mathrm{cm})$ & $102( \pm 6.4)$ & $102( \pm 6.3)$ & $102( \pm 5.5)$ & $102( \pm 5.9)$ & 0.944 \\
\hline Weight (kg) & $70( \pm 12.1)$ & $70( \pm 12.1)$ & $71( \pm 11.6)$ & $70( \pm 11.6)$ & 0.974 \\
\hline BMI $\left(\mathrm{kg} / \mathrm{m}^{2}\right)$ & $24( \pm 3.4)$ & $24( \pm 3.5)$ & $24( \pm 3.0)$ & $24( \pm 3.0)$ & 0.956 \\
\hline $\mathrm{TC}(\mathrm{mg} / \mathrm{dl})$ & $204( \pm 22.0)$ & $202( \pm 20.7)$ & $217( \pm 22.0)$ & $213( \pm 26.9)$ & 0271 \\
\hline HDL (mg/dl) & $64( \pm 11.7)$ & $64( \pm 11.5)$ & $66( \pm 14.0)$ & $65( \pm 12.8)$ & 0.395 \\
\hline TC/HDL & $3.3( \pm 0.7)$ & $3.2( \pm 0.7)$ & $3.5( \pm 0.8)$ & $3.4( \pm 0.9)$ & 0.592 \\
\hline LDL (mg/dl) & $120( \pm 18.6)$ & $118( \pm 20.4)$ & $129( \pm 21.7)$ & $126( \pm 24.7)$ & 0.378 \\
\hline $\mathrm{TG}(\mathrm{mg} / \mathrm{dl})$ & $92( \pm 32.9)$ & $94( \pm 36.1)$ & $96( \pm 51.3)$ & $95( \pm 49.0)$ & 0.924 \\
\hline Apo A1 (mg/dl) & $144( \pm 15.9)$ & $145( \pm 16.5)$ & $145( \pm 21.3)$ & $144( \pm 17.0)$ & 0.636 \\
\hline Apo B (mg/dl) & $91( \pm 13.8)$ & $91( \pm 15.3)$ & $95( \pm 14.6)$ & $95( \pm 17.7)$ & 0.920 \\
\hline Glu (mg/dl) & $93( \pm 7.0)$ & $93( \pm 6.9)$ & $93( \pm 10.9)$ & $95( \pm 10.8)$ & 0.418 \\
\hline Ins (mcU/ml) & $7.0( \pm 5.3)$ & $6.1( \pm 2.8)$ & $6.7( \pm 5.1)$ & $6.5( \pm 5.0)$ & 0.500 \\
\hline CRP (mg/L) & $1.5( \pm 2.7)$ & $1.4( \pm 2.2)$ & $1.1( \pm 1.0)$ & $1.2( \pm 1.3)$ & 0.964 \\
\hline Ox LDL (U/L) & $59.8( \pm 16.7)$ & $57.3( \pm 17.2)$ & $63.5( \pm 17.2)$ & $64.3( \pm 21.3)$ & 0.634 \\
\hline F2 Iso $(\mu \mathrm{g} / 24 \mathrm{~h})$ & $2.20( \pm 0.90)$ & $2.22( \pm 0.84)$ & $2.04( \pm 0.68)$ & $2.01( \pm 0.94)$ & 0.739 \\
\hline
\end{tabular}

$p$ value is relative to one-way ANOVA on the difference between T0 and T1

SYS systolic pressure, DIA diastolic pressure, $W M$ waist measure, $H M$ hips measure, $B M I$ body mass index, $T C$ plasma total cholesterol, $H D L$ plasma high-density lipoproteins, $L D L$ plasma low-density lipoproteins, $T G$ plasma triglycerides, Apo Apolipoproteins in plasma, Glu plasma glucose, Ins plasma insulin, CRP plasma reactive C protein, oxLDL plasma oxidized LDL, F2 Isp total 24-h urine Isoprostane F2 
spp. [Bif F: TCG CGT C(C/T)G GTG TGA AAG; Bif R: CCA CAT CCA GC(A/G) TCC AC] and for total bacteria (Bact 1369: CGG TGA ATA CGT TCC CGG; Prok 1492 TAC GGC TAC CTT GTT ACG ACTT). Reactions were performed at the specified conditions (see reference) using SsoFAST Evagreen SupemixKit (BIO RAD) and a Lightcycler 480 PCR machine (Roche). Quantifications were done using standard curves obtained by amplifying pure cultures of Bb12 which had been previously quantified by plate counting. For total bacteria a mixture of bacterial DNA was obtained by pooling the total faecal genomic DNA from four faecal samples, which had been previously enumerated using FCM-FISH.

\section{Metabolite analysis}

Targeted metabolomics analysis by UHPLC-ESI-MS/MS was carried out as previously described [30, 34] on 24-h urine and fasted blood samples. After methanol extraction of polyphenols, analysis was performed by an ultra-performance liquid chromatographic system (UHPLC) coupled with a tandem mass spectrometer. The system used was an ACQUITY UPLC system coupled to a Xevo TQ triple quadrupole via an electrospray (ESI) interface (Waters, Milford, MA, USA). The separation was performed with a Waters ACQUITY UPLC column HSS T3 $(100 \mathrm{~mm} \times 2.1 \mathrm{~mm}$, $1.8 \mu \mathrm{m})$ equipped with a guard column. The injection volume was $5 \mu$ l. Mobile phases of $0.1 \%$ formic acid in Milli$\mathrm{Q}$ water (A) and $0.1 \%$ formic acid in acetonitrile (B) were used. Chromatographic separation was performer using a gradient as follows: $0 \mathrm{~min}, 5 \% \mathrm{~B}$; $0-3 \mathrm{~min}, 5-20 \% \mathrm{~B}$; 3-4.30 min, 20\% B; 4.30-9 min, 20-45\% B; 9-11 min, 45-100\% B; 11-14 min, $100 \% \mathrm{~B}$; and 14.01-17 min, 5\% $\mathrm{B}$ as equilibration time. For calibration, a standard mixture of polyphenol metabolites was serially diluted in aqueous methanol (50:50) at a concentration range of $0.01-20 \mathrm{mg} / \mathrm{l}$. Quantitative data were processed withTargetlynx software (Masslynx, Waters).

\section{Statistical analysis}

Statistical analysis was performed using STATISTICA 13.1 statistics software for data analysis. Data were checked for normality using the Kolmogorov-Smirnov and Shapiro-Wilk tests. Treatment effects were assessed using oneway analysis of variance, or non-parametric Mann-Whitney test. Treatments were compared to each other using a paired Student's $t$ test. $p$ values $<0.05$ were deemed statistically significant. qPCR data analysis was performed using factorial ANOVA (factors: treatment, time) with FDR correction. Urine and plasma metabolite data analysis was performed using factorial ANOVA (factors: treatment, time) with FDR correction.

\section{Results}

\section{Study report}

A total of 73 suitable subjects were identified and were accepted onto the trial to begin the dietary intervention. Eight did not finish the study. Two subjects dropped out because of illness not related to the intervention (flue and surgery), one for family-related issues, five did not like the taste of the product and dropped out. Exclusion occurred because of deviation from the protocol: one volunteer declared after finishing the study to have taken antibiotics and two subjects were excluded because they did not consume the product as directed (more than 25\% returned unconsumed). In total 62 people completed the study successfully and were included in the statistical analysis. In detail, 32 females and 30 males, between 30 and 65 years old, with BMI from 20 to 29.9 (average $24 \pm 3.4$ ) and total cholesterol ranging between 180 and $240 \mathrm{mg} / \mathrm{dl}$ completed the study. Female group had an average age of 48 years $( \pm 8.5)$, while the male group average age was $49( \pm 9.6)$. Between the treatment groups at baseline, blood pressure (average 120/74 and 122/74), BMI (average 24 and 24) and total cholesterol (average 204 and 217), no significant differences were measured (Table 2).

\section{Faecal microbiota analysis}

\section{S rRNA gene community analysis}

A total of $8,924,305$ reads was generated, with an average of $68,648.5 \pm 36,038.2$, mean \pm SD, high-quality $16 \mathrm{~S}$ rRNA gene sequences per stool sample. Microbiota diversity was evaluated for alpha diversity (diversity within a sample, Chao index) and beta diversity (diversity between samples, Bray-Curtis dissimilarity) using QIIME (https://www.qiime. org, [35]). Sequences with expected error rate $>1.5 \%$ and length $<400 \mathrm{bp}$ were removed from analysis. After filtering and chimera removal, $1418.2 \pm 623.9$, mean $\pm S D$, Operational Taxonomic Units (OTU) were obtained on average per sample analysed. OTUs that were present in less than $25 \%$ of the samples were removed.

The two intervention treatments did not show statistically significant differences in alpha diversity $(2634.54 \pm 943.69$ vs $2682.012 \pm 1177.8$ and $2419.24 \pm 1170.06$ vs $2753.09 \pm 875.9$ Chao index, V1 vs V2, OEP and CTRL, respectively; $p=0.29)$ and beta diversity $(0.433 \pm 0.087 \mathrm{vs}$ $0.43 \pm 0.064$ Bray-Curtis dissimilarity index, mean $\pm S D$, OEP vs CTRL, respectively; $p=0.66$ ) (Fig. 2a, b).

At the phylum level, although there was a trend towards increased Actinobacteria with both treatments, there was no statistically significant change within or between treatments over the course of the trial (Fig. 3). 
Fig. 2 Alpha- (a) and betadiversity (b) indexes after $16 \mathrm{~S}$ rRNA metagenomic analysis of faecal samples collected before (T0) and after (T1) dietary intervention. Center lines show the medians; box limits indicate the 25 th and 75 th percentiles as determined by R software; whiskers extend 15 times the interquartile range from the 25th and 75th percentiles; outliers are represented by dots

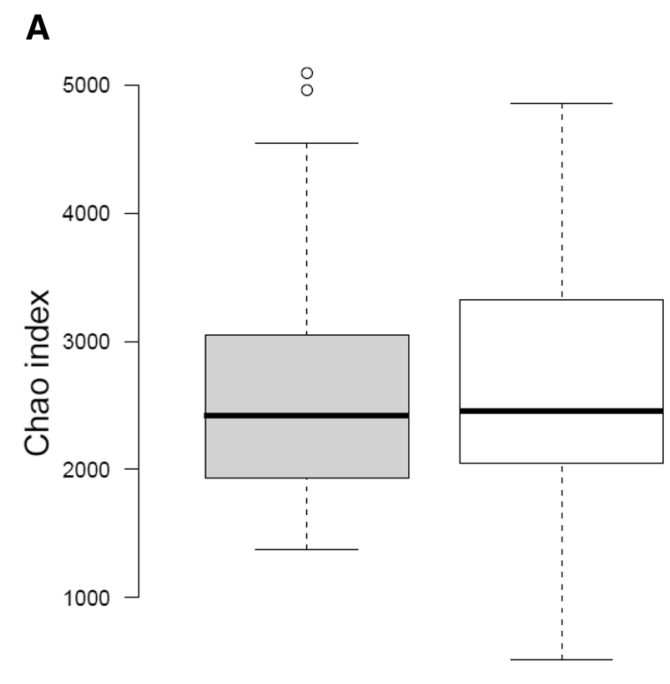

OEP

B

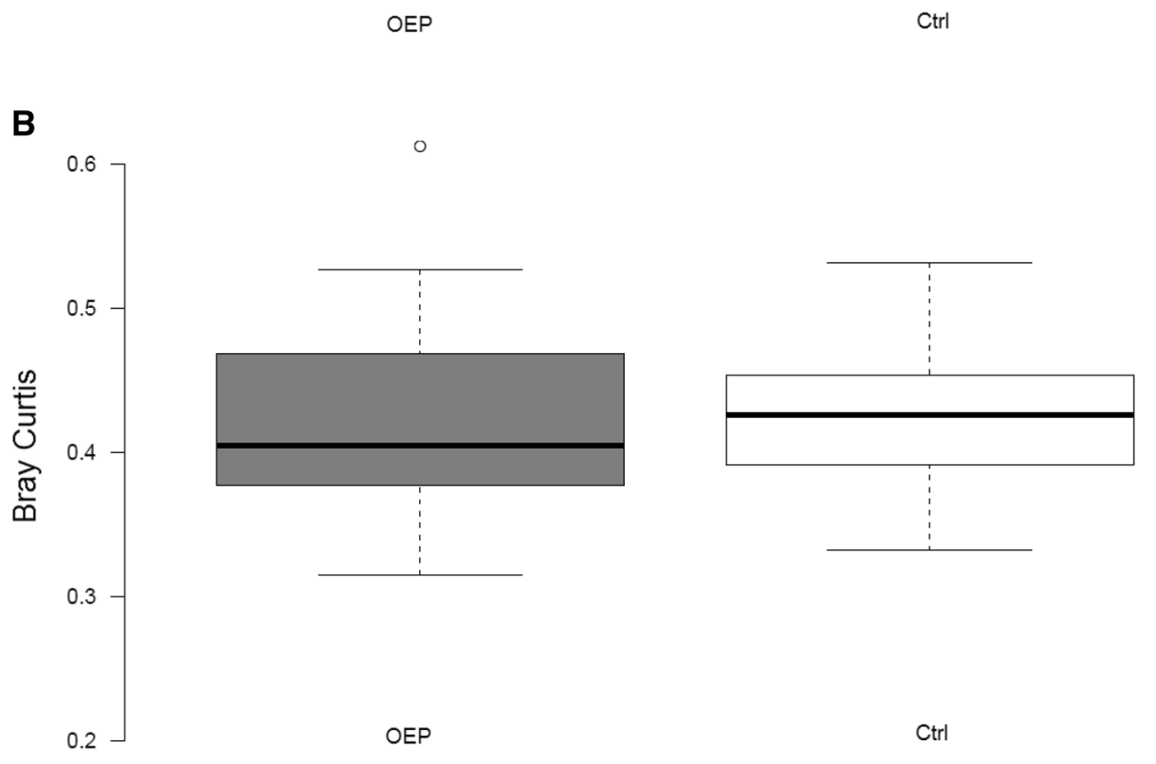

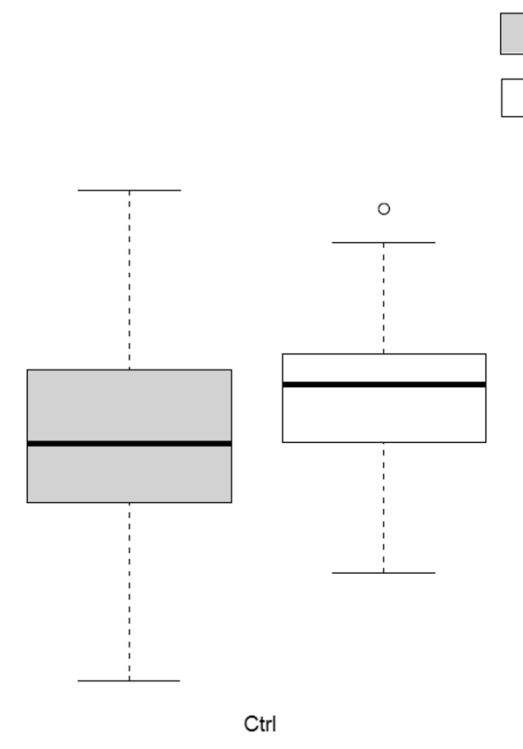

TO

T1
At a genus taxonomic level, a significant although very small increase of Lactobacillus and Ruminococcus was observed after intervention with CTRL compared to OEP $(0.22 \pm 1.21$ and $0.27 \pm 1.45$ vs $0.07 \pm 0.33$ and $0.05 \pm 0.31$, $p=0.042$ for Lactobacillus; $0.54 \pm 0.60$ and $0.70 \pm 0.76$ vs $0.77 \pm 0.93$ and $0.43 \pm 0.38$ for Ruminococcus, $p=0.0191$, percentage relative abundance, V1 and V2, CTRL vs OEP, respectively). Also very small changes in relative abundance of the less dominant bacterial genera were observed $(0.0007 \pm 0.002$ and $0.0009 \pm 0.001$ vs $0.0011 \pm 0.002$ and $0.0003 \pm 0.0007$, for an unknown genus Gemellaceae family, $p=0.017$; and $0.0011 \pm 0.0026$ and $0.0047 \pm 0.0102$ vs $0.0015 \pm 0.0031$ and $0.0011 \pm 0.0023, p=0.032$ for Anaerofustis, percentage relative abundance, V1 and V2, CTRL vs OEP, respectively). Figure 3 a shows the change in relative abundance at genus level for Bifidobacterium, Ruminococcus and Lactobacillus for OEP and CTRL treatments between $\mathrm{V} 1$ and V2.
Culture-independent targeted quantitative enumeration of faecal bacteria

No significant differences were observed between treatment OEP and CTRL compared to baseline values $(1.85 \pm 2.89$ and $2.17 \pm 3.33$ vs $1.39 \pm 1.70$ and $2.20 \pm 3.88)$ for Bifidobacterium spp. $(1.07 \pm 1.57$ and $1.06 \pm 1.31$ vs $0.53 \pm 0.78$ and $0.61 \pm 1.11$ ) for Lactobacillus/Enterococcus spp. $(0.58 \pm 0.97$ and $0.54 \pm 0.54$ vs $0.50 \pm 0.79$ and $0.59 \pm 0.87$, for Ruminococcus obeum group V1 and V2, OEP vs CTRL, respectively, $p>0.05$ factorial ANOVA). Figure 4 shows the $\%$ of bacteria enumerated with oligonucleotidic probes specific for Bifidobacterium spp., Lactobacillus/Enterococcus spp. and Ruminococcus spp. 

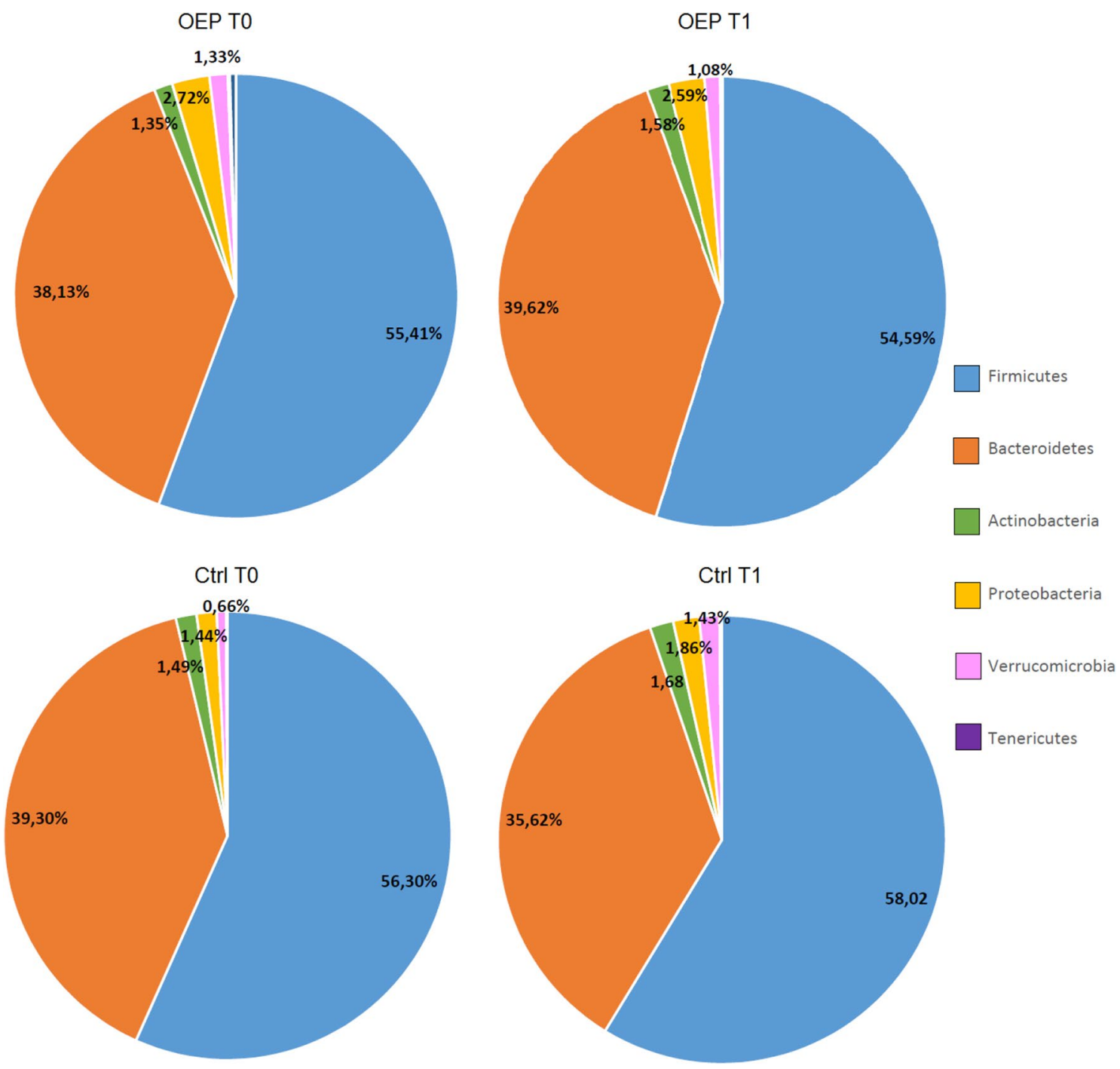

Fig. 3 Percentage relative abundance after 16S rRNA metagenomics analysis of dominant bacterial phyla in faecal samples collected before (T0) and after (T1) dietary intervention with olive-enriched product (OEP) or control product (Ctrl)

\section{Quantitative PCR}

No significant changes in faecal bifidobacteria $(6.34 \pm 0.95$ and $6.62 \pm 0.92$ vs $6.67 \pm 0.74$ and $6.72 \pm 0.74, \mathrm{~V} 1$ and $\mathrm{V} 2$, $\mathrm{P}$ vs $\mathrm{W}$, respectively, $p>0.05$, factorial ANOVA) or in total faecal bacteria $(10.08 \pm 0.04$ and $10.08 \pm 0.05$ vs $10.07 \pm 0.05$ and $10.07 \pm 0.04, \mathrm{~V} 1$ and V2, OEP vs CTRL, respectively, $p>0.05$, factorial ANOVA) were observed after qPCR analysis (data not shown).

\section{Urinary metabolites quantified by LC-MS}

The results of targeted urinary polyphenols are shown in Table 3. Metabolites which were below the detection limit in the majority of samples were excluded from further analysis. Statistical analysis (factorial ANOVA) showed that a number of polyphenol metabolites were significantly higher after OEP treatment compared to CTRL. In particular, 3-3-hydroxyphenyl propanoic acid $(p=0.009)$, 3,4-dihydroxyphenyl acetic acid $(p<0.001)$, hippuric acid $(p=0.014)$, caffeic acid $(p=0.003)$, homovanillic acid $(p<0.001), 3$-hydroxyphenyl acetic acid $(p=0.001)$, sinapic acid $(p=0.002)$, scopoletin $(p=0.001)$. 2,4-Dihydroxybenzoic acid $(p<0.001), 2,5$-dihydroxybenzoic acid $(p=0.022), 3$-(3-hydroxyphenyl) propionic acid $(p=0.009)$, were increased after OEP feeding.

\section{Plasma metabolites quantified by LC-MS}

The results of targeted quantification of plasma metabolites by LC-MS are shown in Table 4. Metabolites which were below the detection limit in the majority of samples 
Fig. 4 Difference in percentage relative abundance of relevant bacterial genera before (T0) and after (T1) dietary intervention with olive-enriched product (OEP) or control product (Ctrl). $p=0.73, p=0.034$ and $p=0.02$, respectively, for Bifidobacterium, Lactobacillus and Ruminococcus genera, after comparison of the difference T1-T0 between olive-enriched product (OEP) and control product (Ctrl), according to Mann-Whitney $U$ test. Center lines show the medians; box limits indicate the 25th and 75th percentiles as determined by $\mathrm{R}$ software; whiskers extend 1.5 times the interquartile range from the 25th and 75th percentiles; outliers are represented by dots

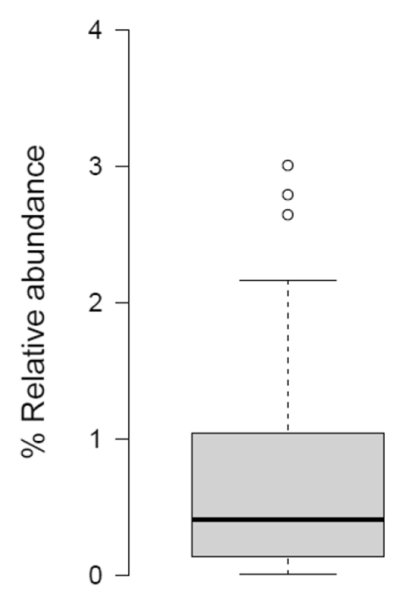

Bifidobacterium

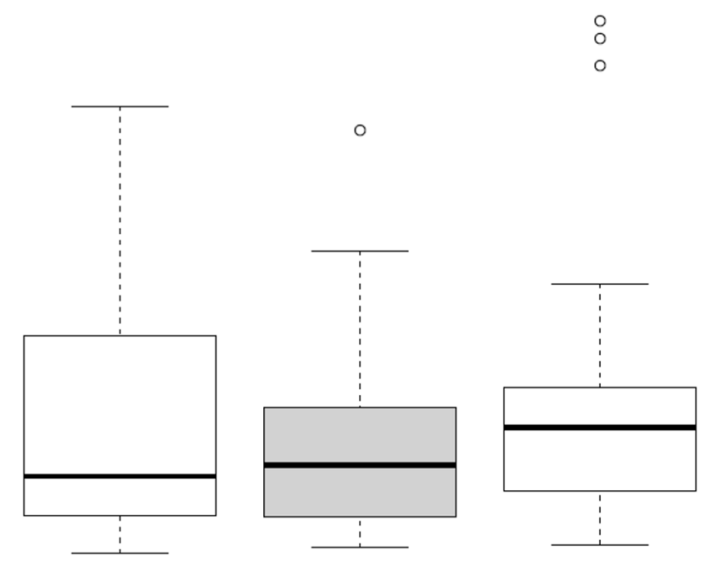

OEP

Ctrl
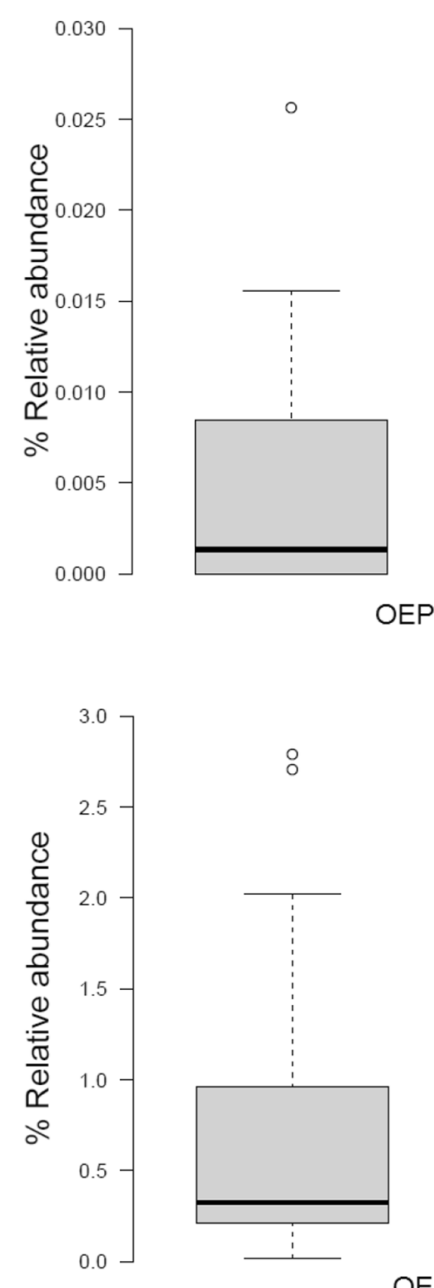

\section{Lactobacillus}

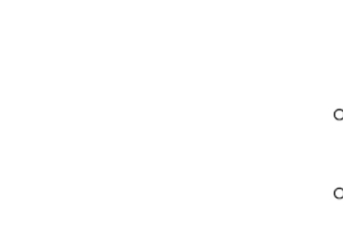

$\circ$
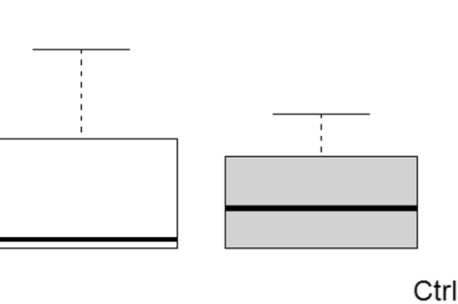

Ctrl

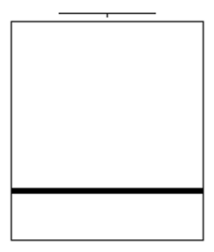

Ruminococcus
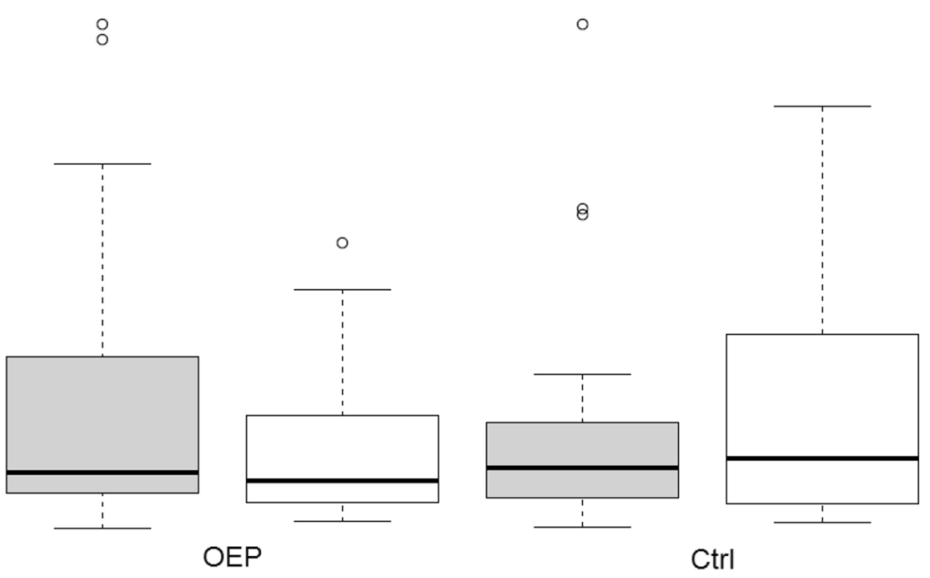

were excluded from further analysis. Statistical analysis (factorial ANOVA) showed that 3,4-dihydroxyphenyl acetic acid $(p=0.002)$ and homovanillic acid $(p=0.003)$ were significantly higher after OEP treatment compared to CTRL. Most of the polyphenol metabolites were present at very low concentrations in plasma compared to urine since the plasma samples were taken in a fasted state. 
Table 3 Urinary polyphenol concentration $(\mu \mathrm{M})$ quantified by mass spectrometry and normalized according to 24 -h urine volume

\begin{tabular}{llllll}
\hline Treatment time & OEP & & & Ctrl & $p$ value \\
\cline { 2 - 3 } & T0 & T1 & T0 & T1 \\
\hline
\end{tabular}

Anthranilic acid $(\mu \mathrm{M})$

Mean

SD $\quad 0.98$

4-Aminobenzoic acid $(\mu \mathrm{M})$

Mean $\quad 0.03$

SD

0.02

Vanillin $(\mu \mathrm{M})$

Mean

SD

66.66

1.38

1.33

0.94

0.86

1.46

0.467

Acetovanillone $(\mu \mathrm{M})$

$\begin{array}{lllll}\text { Mean } & 2.66 & 2.58 & 3.13 & 2.60 \\ \text { SD } & 2.45 & 1.47 & 3.64 & 1.87\end{array}$

2,4-DiOH-benzoic acid $(\mu \mathrm{M})$

$\begin{array}{lll}\text { Mean } & 0.81 & 1.09 \\ \text { SD } & 0.34 & 0.44\end{array}$

3,5-DiOH-benzoic acid $(\mu \mathrm{M})$

Mean $\quad 3.01$

SD $\quad 1.93$

2,5-DiOH-benzoic acid $(\mu \mathrm{M})$

$\begin{array}{ll}\text { Mean } & 2.93 \\ \text { SD } & 2.83\end{array}$

Neochlorogenic acid $(\mu \mathrm{M})$

$\begin{array}{ll}\text { Mean } & 0.43 \\ \text { SD } & 0.43\end{array}$

Chlorogenic acid $(\mu \mathrm{M})$

$\begin{array}{llllll}\text { Mean } & 0.59 & 0.57 & 0.55 & 0.46 & 0.738 \\ \text { SD } & 0.44 & 0.51 & 0.51 & 0.41 & \end{array}$

Fertaric acid $(\mu \mathrm{M})$

$\begin{array}{llllll}\text { Mean } & 0.12 & 0.11 & 0.11 & 0.13 & 0.475 \\ \text { SD } & 0.23 & 0.12 & 0.14 & 0.18 & \end{array}$

$t$-Coutaric acid $(\mu \mathrm{M})$

$\begin{array}{llllll}\text { Mean } & 0.04 & 0.03 & 0.05 & 0.05 & \\ \text { SD } & 0.07 & 0.04 & 0.08 & 0.11 & 0.614\end{array}$

Phloretin $(\mu \mathrm{M})$

Mean

SD

0.04

0.02

Phlorizin $(\mu \mathrm{M})$

Mean

SD

0.07

0.02

$0.05 \quad 0.03$

$0.09 \quad 0.06$

0.214

Naringenin $(\mu \mathrm{M})$

$\begin{array}{lllll}\text { Mean } & 0.07 & 0.04 & 0.10 & 0.08 \\ \text { SD } & 0.08 & 0.03 & 0.11 & 0.10\end{array}$

SD
Phloroglucinol $(\mu \mathrm{M})$

\begin{tabular}{lrrrrr} 
Mean & 15.09 & 15.43 & 15.68 & 15.54 & \\
SD & 5.49 & 5.93 & 6.26 & 6.26 & 0.788 \\
4-Hydroxybenzoic acid $(\mu \mathrm{M})$ & & & & \\
Mean & 6.27 & 6.17 & 6.81 & 5.24 & \\
SD & 3.87 & 4.13 & 3.06 & 2.24 & 0.076 \\
\hline
\end{tabular}

Table 3 (continued)

Treatment time OEP T0 T1

Ctrl

$p$ value

$m$-Coumaric acid $(\mu \mathrm{M})$

$\begin{array}{lllll}\text { Mean } & 0.67 & 0.81 & 0.68 & 0.60\end{array}$

$\begin{array}{lllll}\text { SD } & 0.87 & 0.79 & 0.87 & 0.66\end{array}$

0.341

3-(3-Hydroxyphenyl) propanoic acid $(\mu \mathrm{M})$

$\begin{array}{llllll}\text { Mean } & 20.65 & 34.02 & 16.12 & 13.83 & \\ \text { SD } & 18.12 & 24.58 & 21.20 & 18.33 & 0.009 \\ \text { Vanillic acid }(\mu \mathrm{M}) & & & & & \\ \text { Mean } & 2.80 & 3.16 & 2.32 & 2.25 & \\ \text { SD } & 2.50 & 2.19 & 2.39 & 1.95 & 0.499\end{array}$

3,4-Dihydroxyphenyl acetic acid $(\mu \mathrm{M})$

$\begin{array}{lrrrrr}\text { Mean } & 1.39 & 22.09 & 1.68 & 0.94 & \\ \begin{array}{lrrrr}\text { SD } \\ \text { Hippuric acid }(\mu \mathrm{M})\end{array} & 3.04 & 13.19 & 3.28 & 2.11 & <0.001 \\ \begin{array}{l}\text { Mean } \\ \text { SD }\end{array} & & & & \\ \text { SD } & 277.46 & 331.38 & 321.79 & 313.13 & 0.014 \\ \text { Caffeic acid }(\mu \mathrm{M}) & & & & & \\ \text { Mean } & 0.45 & 0.64 & 0.47 & 0.44 & \\ \text { SD } & 0.34 & 0.28 & 0.24 & 0.24 & 0.003\end{array}$

Homovanillic acid $(\mu \mathrm{M})$

$\begin{array}{lrrrrr}\text { Mean } & 20.96 & 54.41 & 21.63 & 19.62 & \\ \text { SD } & 7.36 & 21.87 & 8.12 & 8.02 & <0.001\end{array}$

Isoferulic acid $(\mu \mathrm{M})$

$\begin{array}{lccccc}\text { Mean } & 1.45 & 1.72 & 0.59 & 0.93 & \\ \text { SD } & 3.88 & 4.62 & 0.61 & 1.76 & 0.577 \\ t \text {-Ferulic acid }(\mu \mathrm{M}) & & & & & \\ \text { Mean } & 2.47 & 2.35 & 1.71 & 3.07 & \\ \text { SD } & 2.62 & 2.40 & 1.17 & 6.01 & 0.901\end{array}$

Alpha-hydroxyhippuric acid $(\mu \mathrm{M})$

$\begin{array}{llllll}\text { Mean } & 0.06 & 0.05 & 0.04 & 0.05 & \\ \text { SD } & 0.07 & 0.05 & 0.05 & 0.06 & 0.829 \\ \text { Urolithin A }(\mu \mathrm{M}) & & & & & \\ \text { Mean } & 0.26 & 0.23 & 0.49 & 0.36 & \\ \text { SD } & 0.40 & 0.58 & 1.11 & 0.86 & 0.623\end{array}$

3-Hydroxyphenyl acetic acid $(\mu \mathrm{M})$

$\begin{array}{llllll}\text { Mean } & 34.82 & 65.11 & 28.76 & 29.58 & \\ \text { SD } & 40.05 & 43.29 & 18.77 & 27.69 & 0.001\end{array}$

Hydroferulic acid $(\mu \mathrm{M})$

\begin{tabular}{lccccc} 
Mean & 3.95 & 5.47 & 4.87 & 3.82 & \\
SD & 5.20 & 5.21 & 5.19 & 3.71 & 0.106 \\
Sinapic acid $(\mu \mathrm{M})$ & & & & & \\
$\quad$ Mean & 0.64 & 1.06 & 0.78 & 0.67 & \\
SD & 0.51 & 0.78 & 0.71 & 0.69 & 0.002 \\
Protocatechuic acid $(\mu \mathrm{M})$ & & & & \\
Mean & 0.55 & 0.75 & 0.54 & 0.50 & \\
SD & 0.59 & 0.39 & 0.32 & 0.28 & 0.001 \\
Scopoletin $(\mu \mathrm{M})$ & & & & & \\
Mean & 0.14 & 0.29 & 0.11 & 0.13 & \\
SD & 0.14 & 0.16 & 0.12 & 0.15 & 0.001 \\
\hline
\end{tabular}


Table 3 (continued)

\begin{tabular}{|c|c|c|c|c|c|}
\hline \multirow[t]{2}{*}{ Treatment time } & \multicolumn{2}{|l|}{ OEP } & \multicolumn{2}{|l|}{ Ctrl } & \multirow[t]{2}{*}{$p$ value } \\
\hline & T0 & $\mathrm{T} 1$ & T0 & $\mathrm{T} 1$ & \\
\hline \multicolumn{6}{|c|}{ Cryptochlorogenic acid $(\mu \mathrm{M})$} \\
\hline Mean & 0.15 & 0.14 & 0.12 & 0.14 & \\
\hline SD & 0.20 & 0.15 & 0.15 & 0.19 & 0.880 \\
\hline \multicolumn{6}{|c|}{ Syringic acid $(\mu \mathrm{M})$} \\
\hline Mean & 0.48 & 0.64 & 0.62 & 0.59 & \\
\hline SD & 0.27 & 0.49 & 0.50 & 0.47 & 0.083 \\
\hline \multicolumn{6}{|c|}{ 4-Hydroxyphenyl acetic acid $(\mu \mathrm{M})$} \\
\hline Mean & 109.73 & 125.51 & 96.81 & 101.87 & \\
\hline SD & 86.90 & 65.10 & 40.88 & 57.97 & 0.290 \\
\hline
\end{tabular}

Data represent the mean and standard deviation (SD) at the beginning (T0) and at the end (T1) of dietary intervention with olive-enriched product (OEP) or control product (Ctrl), and the relative $p$ value after factorial ANOVA with Bonferroni's correction. Cinnamic acid, caftaric acid, cis-piceide, luteolin, hesperidin, catechin, epicatechin, procyanidin B1, procyanidin B2+B4, procyanidin B3, quercetin-3-Rha, kaempferol-3-Glc, kaempferol-3-rutinoside, dihydrokaempferol, quercetin-3-glucuronide, kaempferol-3-glucuronide, arbutin, $p$-coumaric acid, $o$-coumaric acid, gallic acid, ellagic acid, pyrocatechol, urolithin B, epigallocatechin gallate, epicatechin gallate, quercetin3-Glc + quercetin-3-gal, isorhamnetin-3-Glc, rutin, salidroside are not shown, since the levels fell below limit of quantification

\section{Clinical measures of CVD risk and inflammation}

Subjects in either group, OEP or CTRL, were matched for age and sex. Little difference was observed in baseline clinical parameters between the groups before dietary intervention. After 8 weeks of treatment with either biscuit, no significant change in CVD or inflammatory makers was observed (Table 2). There was a trend towards reduced oxidized LDL cholesterol in the OEP-treated group, but this was not significant either with respect to time or compared to the control treatment.

\section{Sex impacted on urinary metabolite profiles}

Although sex did not appear to influence to response to OEP ingestion in urine or in plasma, differences were observed in the concentrations of small phenolic acids excreted by women compared to men after the OEP treatment. Higher amounts of 3,5-diOH-benzoic acid, $t$-coutaric acid, naringenin, 4-hydroxybenzoic acid, 4-hydroxyphenyl acetic acid were excreted by male subjects compared to female subjects after the OEP treatment (Table 5). Since the gut microbiota is intricately involved in the metabolism of complex polyphenolic compounds and especially in the production of small phenolic acids, we measured whether the gut microbiota of male and female subjects differed after OEP ingestion. Statistically significant differences in the relative abundance of Akkermansia, Bifidobacterium, Bacteroides, Prevotella,
Table 4 Plasma polyphenol concentration $(\mu \mathrm{M})$ quantified by mass spectrometry

\begin{tabular}{lllllll}
\hline Treatment time & OEP & & & Ctrl & $p$ value \\
\cline { 2 - 3 } & T0 & T1 & & T1 & \\
\hline
\end{tabular}

Anthranilic acid $(\mu \mathrm{M})$

$\begin{array}{lccccc}\text { Mean } & 0.013 & 0.018 & 0.016 & 0.016 & 0.709 \\ \text { SD } & 0.012 & 0.023 & 0.015 & 0.015 & \\ \text { Vanillin }(\mu \mathrm{M}) & & & & & \\ \quad \text { Mean } & 0.092 & 0.087 & 0.078 & 0.065 & 0.614 \\ \text { SD } & 0.090 & 0.058 & 0.042 & 0.030 & \\ \text { 2,4-DiOH-benzoic acid }(\mu \mathrm{M}) & & & & \\ \text { Mean } & 0.006 & 0.008 & 0.007 & 0.005 & 0.891 \\ \text { SD } & 0.009 & 0.011 & 0.013 & 0.008 & \\ \text { Phlorizin }(\mu \mathrm{M}) & & & & & \\ \quad \begin{array}{l}\text { Mean } \\ \text { SD }\end{array} & 0.006 & 0.009 & 0.005 & 0.021 & 0.974 \\ \text { Pyrogallol }(\mu \mathrm{M}) & 0.008 & 0.028 & 0.007 & 0.080 & \\ \quad & & & & & \\ \text { Mean } & 0.182 & 0.157 & 0.238 & 0.235 & 0.738 \\ \text { SD } & 0.319 & 0.357 & 0.584 & 0.465 & \end{array}$

4-Hydroxybenzoic acid $(\mu \mathrm{M})$

$\begin{array}{llllll}\text { Mean } & 0.217 & 0.046 & 0.274 & 0.094 & 0.798 \\ \text { SD } & 0.303 & 0.086 & 0.301 & 0.166 & \end{array}$

3-(3-Hydroxyphenyl propanoic acid $(\mu \mathrm{M})$

$\begin{array}{llllll}\text { Mean } & 1.449 & 1.614 & 2.286 & 1.735 & 0.551 \\ \text { SD } & 0.784 & 1.292 & 3.646 & 1.098 & \end{array}$

Vanillic acid $(\mu \mathrm{M})$

$\begin{array}{llllll}\text { Mean } & 0.041 & 0.033 & 0.041 & 0.055 & 0.689 \\ \text { SD } & 0.028 & 0.020 & 0.025 & 0.099 & \end{array}$

3,4-Dihydroxyphenyl acetic acid $(\mu \mathrm{M})$

$\begin{array}{llllll}\text { Mean } & 0.010 & 0.651 & 0.006 & 0.021 & <0.001 \\ \text { SD } & 0.032 & 0.493 & 0.031 & 0.062 & \end{array}$

Hippuric acid $(\mu \mathrm{M})$

$\begin{array}{lrrrrr}\text { Mean } & 11.200 & 14.524 & 13.034 & 14.003 & 0.355 \\ \text { SD } & 7.438 & 9.166 & 10.503 & 11.230 & \end{array}$

Caffeic acid $(\mu \mathrm{M})$

$\begin{array}{llllll}\text { Mean } & 0.024 & 0.018 & 0.013 & 0.015 & 0.651\end{array}$

$\begin{array}{lllll}\text { SD } & 0.045 & 0.023 & 0.016 & 0.018\end{array}$

Homovanillic acid $(\mu \mathrm{M})$

$\begin{array}{llllll}\text { Mean } & 0.093 & 0.217 & 0.069 & 0.090 & 0.003 \\ \text { SD } & 0.075 & 0.135 & 0.061 & 0.057 & \\ \text { Syringic acid }(\mu \mathrm{M}) & & & & & \\ \text { Mean } & 0.015 & 0.007 & 0.012 & 0.032 & 0.093 \\ \text { SD } & 0.021 & 0.014 & 0.016 & 0.109 & \end{array}$

4-Hydroxyphenyl acetic acid $(\mu \mathrm{M})$

\begin{tabular}{llllll} 
Mean & 2.327 & 1.449 & 1.140 & 1.232 & 0.525 \\
SD & 5.450 & 1.521 & 1.131 & 1.209 & 0.709 \\
\hline
\end{tabular}

Data represent the mean and standard deviation (SD) at the beginning (T0) and at the end (T1) of dietary intervention with olive-enriched product (OEP) or control product (Ctrl), and the relative $p$ value after factorial ANOVA with Bonferroni's correction

Chlorogenic acid, cis-piceide, quercetin-3-Rha, quercetin-3-glucuronide, kaempferol-3-glucuronide, $t$-ferulic acid, ellagic acid, protocate- 
Table 4 (continued)

chuic acid, cryptochlorogenic acid, quercetin-3-Glc + quercetin-3-gal, hydroxytyrosol and tyrosol are not shown, since the levels fell below limit of quantification

Table 5 Total urine polyphenols that appeared significantly different between male $(\mathrm{M})$ and female $(\mathrm{F})$ volunteers at the end of intervention (T1) with olive-enriched product (OEP)

\begin{tabular}{lccc}
\hline Treatment time & \multicolumn{2}{c}{ OEP } & \multirow{2}{*}{$p$ value } \\
\cline { 2 - 3 } & $\mathrm{T} 1$ & \\
\cline { 2 - 3 } Gender & $\mathrm{M}$ & $\mathrm{F}$ & \\
\hline 3,5-DiOH-benzoic acid $(\mu \mathrm{M})$ & & \\
Mean & 2.89 & 1.80 & 0.007 \\
SD & 1.44 & 1.65 & \\
$t$-Coutaric acid $(\mu \mathrm{M})$ & & & \\
Mean & 0.04 & 0.02 & 0.021 \\
SD & 0.04 & 0.04 & \\
Naringenin $(\mu \mathrm{M})$ & & & \\
Mean & 0.05 & 0.03 & 0.015 \\
SD & 0.03 & 0.03 & \\
4-Hydroxybenzoic acid $(\mu \mathrm{M})$ & & 0.023 \\
Mean & 6.68 & 5.81 & \\
SD & 2.19 & 5.12 & \\
4-Hydroxyphenyl acetic acid $(\mu \mathrm{M})$ & & \\
Mean & 153.66 & 105.41 & \\
SD & 80.96 & 42.55 & \\
\hline
\end{tabular}

Table shows the $p$ values after factorial ANOVA with Bonferroni's correction. Data represent mean and standard deviation (SD) of urinary concentrations $(\mu \mathrm{M})$, after normalization according to 24 -h urine volume

Rikenellaceae, Barnesiellaceae, and Enterobacteriaceae were observed between the faecal microbiota of men and women (Fig. 5). These differences were statistically significant after correction for repeated measures (Mann-Whitney $U$ test, $2 * 1$ exact $p$ value) (Fig. 6).

\section{Discussion}

The primary objective of this study was to measure the impact of an olive pomace-enriched product (OEP) on the composition and metabolic output of the human gut microbiota. Considering the accepted physiological relevance of olive polyphenols, their apparent ability to protect LDL cholesterol particles from oxidative damage, and the fact that the gut microbiota appears to be intimately related to their metabolism in vivo, we measured changes in key olive-derived polyphenols, including tyrosol and HT, and their derived catabolites using a quantitative LC-MS-based strategy. The OEP biscuits did not have a major impact on the composition of the gut microbiota, but did induce subtle changes in relative abundances of certain bacteria. Significant differences in relative abundance of Lactobacillus, Ruminococcus, Gemellaceae and Anaerofustis were observed between treatments using community level $16 \mathrm{~S}$ rRNA profiling. More quantitative analysis using flow cytometry-coupled fluorescent in situ hybridization did not confirm statistically significance for bifidobacteria, lactobacilli or the Ruminococcus obeum-like bacteria. However, a trend was apparent, consistent between 16S rRNA gene sequencing, the probe-based FISH and qPCR, showing a small increase in bifidobacteria.

In terms of metabolic output, LC-MS-based targeted metabolomics confirmed that ingestion of the OEP biscuits resulted in a significant increase in urinary excretion of small phenolic acids derived from the metabolism of olive polyphenols. These small phenolic acids derive from the combined activities of human phase I and II biotransformation and the action of the gut microbiota. OEP ingestion resulted in a significant increase in excretion of homovanillic acid, 3,4-dihydroxyphenyl acetic acid, scopoletin, protocatechuic acid, sinapic acid, 3-hydroxyphenyl acetic acid, isoferulic acid, caffeic acid, hippuric acid, 3,3-hydroxyphenyl acetic acid, 2,5-dihydroxybenzoic acid and 2,4-dihydroxybenzoic acid. Many of these compounds derive from the breakdown pathways of the tyrosol group enriched in olives and/or the hippuric acid pathway, a pathway common to many classes of polyphenols. Both involve steps mediated by the gut microbiota and these catabolites and similar small phenolic acids have been reported to be excreted following ingestion of olive or olive fractions in previous studies [20-22, 36, 37]. Few studies have reported the profile of metabolites present in fasted blood samples after chronic ingestion of olive pomace. Here we found that ingestion of the OEP biscuits for 8 weeks resulted in a significantly higher level of tyrosol metabolites, homovanillic acid and 3,4-dihydroxyphenyl acetic acid (DOPAC). Homovanillic acid increased more then twofold in the OEP-treated group and was also about double the concentration after the CTRL treatment. For DOPAC, OEP induced more than tenfold increase in fasted blood concentrations, and a similar difference in magnitude was observed compared to the control group. As well as being associated with the protection of LDL cholesterol particles from oxidative damage, both homovanillic acid and DOPAC have been reported to transiently associate with LDL cholesterol particles in blood thereby mediating their antioxidative effect. While most studies report these molecules to be relatively rapidly cleared from the blood [38], our study is one of the very few to record high levels of these antioxidants in fasted blood samples, many hours after ingestion of the olive-enriched food. This is an important observation for the possible functional activities of the OEP biscuit since persistence of these tyrosol-derived metabolites 
Fig. 5 Bacterial populations enumerated by FCM-FISH ( $\%$ of total bacteria enumerated by SYBR green staining) in faecal samples collected before (T0) and after (T1) dietary intervention with olive-enriched product (OEP) or control product (Ctrl). Center lines show the medians; box limits indicate the 25th and 75th percentiles as determined by R software; whiskers extend 1.5 times the interquartile range from the 25 th and 75 th percentiles; outliers are represented by dots

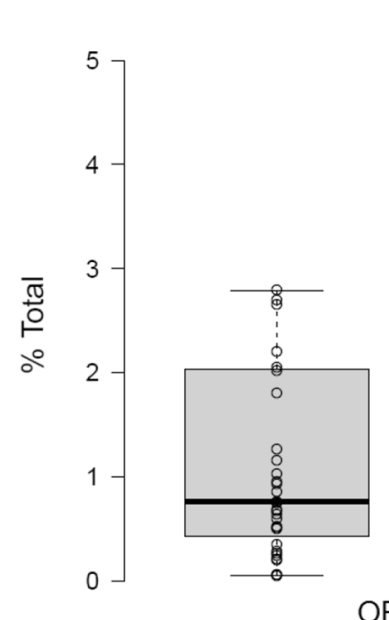

Bifidobacterium spp

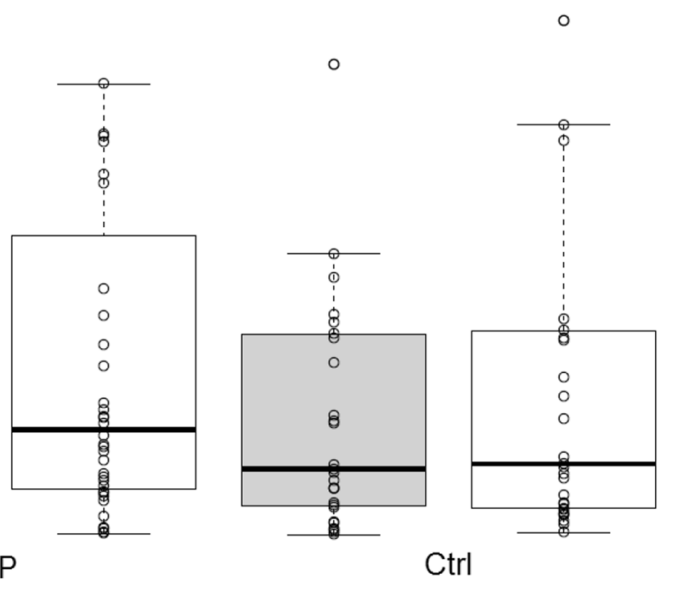

Lactobacillus/Enterococcus spp

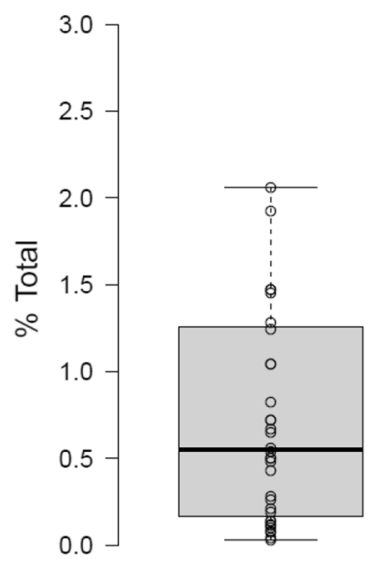

0
0
0
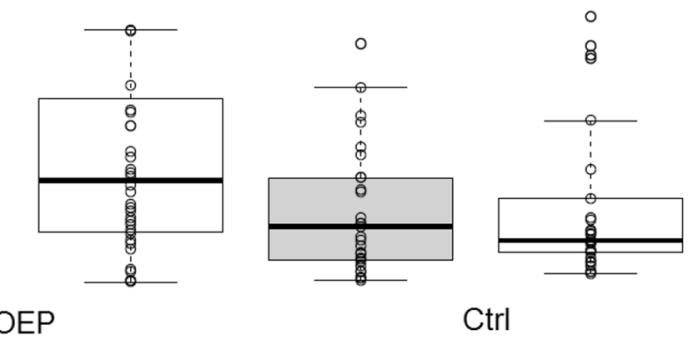

Ctrl

\section{Ruminococcus spp}

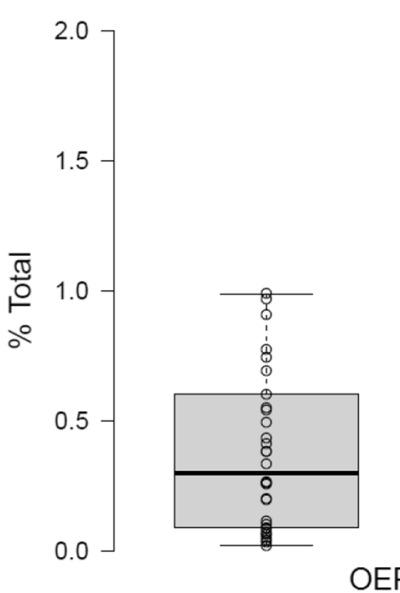

TO $\mathrm{T} 1$ in blood, or indeed within the intestine, could prime antioxidant defences and/or absorption of oxidized LDL cholesterol upon fat meal challenge. However, our current study was not designed to measure the effect of olive polyphenols on oxidized LDL cholesterol levels and therefore, any potential beneficial effect of the OEP biscuit awaits confirmation in an acute, postprandial study. Similarly, homovanillic acid and DOPAC are both involved in the dopamine pathway and have been shown to mediate other physiological effects including ameliorating age-related decline in muscle 

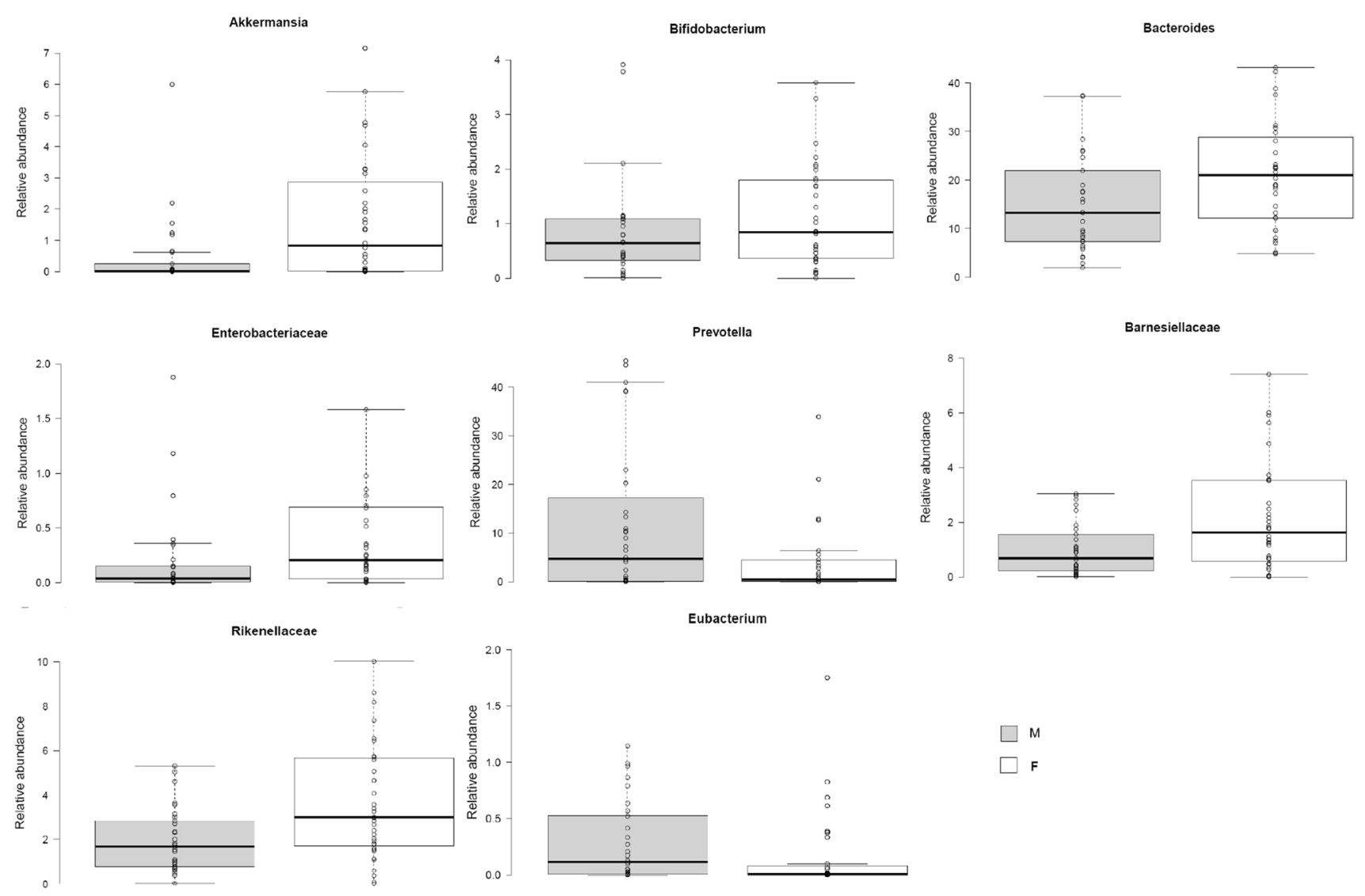

ubacterium

Fig. 6 Significant differences between male (M) and female (F) in percentage relative abundance of bacterial genera after dietary intervention with olive-enriched product (OEP), according to MannWhitney $U$ test. Center lines show the medians; box limits indicate the 25 th and 75 th percentiles as determined by $\mathrm{R}$ software; whiskers extend 15 times the interquartile range from the 25th and 75th percentiles; outliers are represented by dots function [39] and brain function [40]. In addition, DOPAC has been shown to impact on the inflammatory response of immune cells to lipopolysaccharide or endotoxin, an inflammatory microbially derived signal associated with increased risk of metabolic disease [41]. However, any ability of the OEP biscuits to mediate such health effects awaits specifically and appropriately designed human studies.

In this current study, we also measured the ability of the OEP biscuit to modulate blood lipid profiles. Previous studies with whole plant foods or oat-derived beta-glucan in particular, have shown significant and clinically meaningful reductions in cholesterol upon ingestion [42]. However, the mechanisms by which these foods mediate their cholesterol lowering effects are still very much unclear, with different mechanisms suggesting involving phytosterols, gel-forming and cholesterol binding activities, modified bile acid profiles and/or prebiotic type modulation of the gut microbiota [43, 44]. Our OEP, not containing the pit pulp of the olive, did not contain large amounts of plant phytosterols, and only had minor impact on microbiota composition. Although in this case, the olive pomace did not change blood lipid profiles, further studies, possibly with larger sample size be warranted, especially since the ability of olive and olive extracts in general to modulate blood lipid profiles remains to be convincingly established as per the EFSA statement on olive polyphenol extract health claims [18].

The quantities of small phenolic acids in urine differed between men and women upon OEP ingestion. Men excreted significantly more 3,5-diOH-benzoic acid, $t$-coutaric acid, naringenin, 4-hydroxybenzoic acid, 4-hydroxyphenyl acetic acid than women. A sex bias in polyphenol metabolism has been reported previously. Zamora-Ros et al. [45] analysing the EPIC cohort study reported a significant sex bias in excretion of dietary polyphenols. Moreover, the sex differences in dietary polyphenol metabolism also appears to be reflected in the concentration and profiles of polyphenols or their derivatives in different tissues and organs, as shown for grape seed flavanols in rats [46]. Such sex effects could have important implications for the biological activity of these compounds, especially since a sex effect has also been reported in response to dietary interventions measuring physiological change and/or reduced risk of chronic disease 
upon intervention with polyphenol-rich foods [47]. The contribution of the intestinal microbiota to any sex-specific "metabotype" in terms of polyphenol metabolism remains very much unexplored. In our study, faecal samples collected from men and women post-OEP ingestion differed significantly in the relative abundance of Akkermansia, Bifidobacterium, Bacteroides, Prevotella, Rikenellaceae, Barnesiellaceae, and Enterobacteriaceae. Some of these bacteria are linked to host physiology and protection from metabolic and cardiovascular disease (Akkermansia and Bifidobacterium in particular), but also Bacteroides and Prevotella in relation to obesity and traditional dietary paradigms [48-50]. Similarly, the enterobacteria, which includes many intestinal pathogens, appear particularly susceptible to the antibacterial activities of polyphenols [51]. The role of the human gut microbiota in determining the profile and quantities of different polyphenol breakdown products, their bioavailability, bioactivity and nutri-kinetics may constitute and important new compounding factor to be taken into consideration when designing human dietary interventions where polyphenols are considered mediators of physiological effect. Further data from similar human studies are required to confirm the involvement of the gut microbiota in gender-specific polyphenol metabolism and possible implications sex-specific response to diet.

In conclusion, ingestion olive pomace extract-enriched biscuits mediated small changes within the composition of the gut microbiota. Delivering $17.1 \pm 4.01 \mathrm{mg} / 100 \mathrm{~g}$ HT and its derivatives, the OEP biscuits induced a significant increase in excretion of small phenolic acids in urine, indicative of up-regulation of microbial polyphenol biotransformation in the intestine. Quantities of some small phenolic acids differed in urine of men and women, as did relative abundances of important members of the gut microbiota. OEP also led to a significant increase in homovanillic acid and DOPAC in fasted plasma samples, indicating related clearance of these compounds from the blood or extended release and uptake from the intestine. In either case, higher levels of these biologically active compounds mediated by OEP ingestion warrant further investigation in acute or postprandial studies specifically targeting LDL cholesterol oxidation and cognitive function.

Open Access This article is distributed under the terms of the Creative Commons Attribution 4.0 International License (http://creativecommons.org/licenses/by/4.0/), which permits unrestricted use, distribution, and reproduction in any medium, provided you give appropriate credit to the original author(s) and the source, provide a link to the Creative Commons license, and indicate if changes were made.

\section{References}

1. Bonaccio M, Castelnuovo AD, Bonanni A et al (2013) Adherence to a Mediterranean diet is associated with a better healthrelated quality of life: a possible role of high dietary antioxidant content. BMJ Open 3:e003003. https://doi.org/10.1136/ bmjopen-2013-003003

2. Estruch R, Ros E, Salas-Salvadó J et al (2013) Primary prevention of cardiovascular disease with a mediterranean diet. N Engl J Med 368:1279-1290. https://doi.org/10.1056/NEJMoa1200303

3. Tresserra-Rimbau A, Rimm EB, Medina-Remón A et al (2014) Inverse association between habitual polyphenol intake and incidence of cardiovascular events in the PREDIMED study. Nutr Metab Cardiovasc Dis 24:639-647. https://doi.org/10.1016/j. numecd.2013.12.014

4. Susalit E, Agus N, Effendi I et al (2011) Olive (Olea europaea) leaf extract effective in patients with stage- 1 hypertension: comparison with Captopril. Phytomed Int J Phytother Phytopharm 18:251-258. https://doi.org/10.1016/j.phymed.2010.08.016

5. Perrinjaquet-Moccetti T, Busjahn A, Schmidlin C et al (2008) Food supplementation with an olive (Olea europaea L.) leaf extract reduces blood pressure in borderline hypertensive monozygotic twins. Phytother Res 22:1239-1242. https://doi.org/10.1002/ ptr.2455

6. El SN, Karakaya S (2009) Olive tree (Olea europaea) leaves: potential beneficial effects on human health. Nutr Rev 67:632638. https://doi.org/10.1111/j.1753-4887.2009.00248.x

7. Tousoulis D, Papageorgiou N, Antoniades C et al (2010) Acute effects of different types of oil consumption on endothelial function, oxidative stress status and vascular inflammation in healthy volunteers. Br J Nutr 103:43-49. https://doi.org/10.1017/ S0007114509991346

8. Rondanelli M, Giacosa A, Morazzoni P et al (2016) MediterrAsian diet products that could raise HDL-cholesterol: a systematic review. BioMed Res Int. https://doi.org/10.1155/2016/2025687

9. Lockyer S, Rowland I, Spencer JPE et al (2016) Impact of phenolic-rich olive leaf extract on blood pressure, plasma lipids and inflammatory markers: a randomised controlled trial. Eur J Nutr. https://doi.org/10.1007/s00394-016-1188-y

10. Hernáez Á, Fernández-Castillejo S, Farràs M et al (2014) Olive oil polyphenols enhance high-density lipoprotein function in humans: a randomized controlled trial. Arterioscler Thromb Vasc Biol 34:2115-2119. https://doi.org/10.1161/ATVBAHA.114.303374

11. Pais P, Villar A, Rull S (2016) Impact of a proprietary standardized olive fruit extract (SOFE) on cardio-ankle vascular index, visual analog Scale and C-reactive protein assessments in subjects with arterial stiffness risk. Drugs RD 16:355-368. https:// doi.org/10.1007/s40268-016-0147-7

12. Kominami M, Yasutake Y, Ono Y, Shibata H (2014) Supplementation of olive and grape seed extracts improves vascular function in healthy humans: a randomized controlled study. Atherosclerosis 235:e80. https://doi.org/10.1016/j.atherosclerosis.2014.05.207

13. Violi F, Loffredo L, Pignatelli P et al (2015) Extra virgin olive oil use is associated with improved post-prandial blood glucose and LDL cholesterol in healthy subjects. Nutr Diabetes 5:e172. https:// doi.org/10.1038/nutd.2015.23

14. Moreno-Luna R, Muñoz-Hernandez R, Miranda ML et al (2012) Olive oil polyphenols decrease blood pressure and improve endothelial function in young women with mild hypertension. Am J Hypertens 25:1299-1304. https://doi.org/10.1038/ajh.2012.128

15. Widmer R, Freund M, Flammer A et al (2013) Beneficial effects of polyphenol-rich olive oil in patients with early atherosclerosis. Eur J Nutr 52:1223-1231. https://doi.org/10.1007/s00394-012-0433-2

16. Storniolo CE, Roselló-Catafau J, Pintó X et al (2014) Polyphenol fraction of extra virgin olive oil protects against endothelial 
dysfunction induced by high glucose and free fatty acids through modulation of nitric oxide and endothelin-1. Redox Biol 2:971977. https://doi.org/10.1016/j.redox.2014.07.001

17. Wong RHX, Garg ML, Wood LG, Howe PRC (2014) Antihypertensive potential of combined extracts of olive leaf, green coffee bean and beetroot: a randomized, double-blind, placebo-controlled crossover trial. Nutrients 6:4881-4894. https://doi.org/10.3390/ nu6114881

18. EFSA Panel on Dietetic Products, Nutrition and Allergies (NDA) (2011) Scientific Opinion on the substantiation of health claims related to polyphenols in olive and protection of LDL particles from oxidative damage (ID 1333, 1638, 1639, 1696, 2865), maintenance of normal blood HDL cholesterol concentrations (ID 1639), mainte: polyphenols in olive related health claims. EFSA J 9:2033. https://doi.org/10.2903/j.efsa.2011.2033

19. Corona G, Tzounis X, Assunta Dessì M et al (2006) The fate of olive oil polyphenols in the gastrointestinal tract: implications of gastric and colonic microflora-dependent biotransformation. Free Radic Res 40:647-658. https://doi. org/10.1080/10715760500373000

20. Mosele JI, Martín-Peláez S, Macià A et al (2014) Faecal microbial metabolism of olive oil phenolic compounds: in vitro and in vivo approaches. Mol Nutr Food Res 58:1809-1819. https:// doi.org/10.1002/mnfr.201400124

21. Martín-Peláez S, Mosele JI, Pizarro N et al (2017) Effect of virgin olive oil and thyme phenolic compounds on blood lipid profile: implications of human gut microbiota. Eur J Nutr 56:119-131. https://doi.org/10.1007/s00394-015-1063-2

22. Domínguez-Perles R, Auñón D, Ferreres F, Gil-Izquierdo A (2017) Gender differences in plasma and urine metabolites from Sprague-Dawley rats after oral administration of normal and high doses of hydroxytyrosol, hydroxytyrosol acetate, and DOPAC. Eur J Nutr 56:215-224. https://doi.org/10.1007/s00394-015-1071-2

23. Di Gioia D, Barberio C, Spagnesi S et al (2002) Characterization of four olive-mill-wastewater indigenous bacterial strains capable of aerobically degrading hydroxylated and methoxylated monocyclic aromatic compounds. Arch Microbiol 178:208-217. https:// doi.org/10.1007/s00203-002-0445-Z

24. Nair N, Markham J, Woodfield R, Spooner-Hart R (2007) Low cost olive waste management by bioremediation technology

25. Roig A, Cayuela ML, Sánchez-Monedero MA (2006) An overview on olive mill wastes and their valorisation methods. Waste Manag 26:960-969. https://doi.org/10.1016/j.wasman.2005.07.024

26. Borja R, Rincón B, Raposo F (2006) Anaerobic biodegradation of two-phase olive mill solid wastes and liquid effluents: kinetic studies and process performance. J Chem Technol Biotechnol 81:1450-1462. https://doi.org/10.1002/jctb.1563

27. Nair N, Markham J (2008) Recycling solid waste from the olive oil extraction process. Australian Government, Rural Industries Research and Development Corporation

28. Tay J, Brinkworth GD, Noakes M et al (2008) Metabolic effects of weight loss on a very-low-carbohydrate diet compared with an isocaloric high-carbohydrate diet in abdominally obese subjects. J Am Coll Cardiol 51:59-67. https://doi.org/10.1016/j. jacc.2007.08.050

29. Dell RB, Holleran S, Ramakrishnan R (2002) Sample size determination. Ilar J 43:207-213

30. Gasperotti M, Masuero D, Guella G et al (2014) Development of a targeted method for twenty-three metabolites related to polyphenol gut microbial metabolism in biological samples, using SPE and UHPLC-ESI-MS/MS. Talanta 128:221-230. https://doi. org/10.1016/j.talanta.2014.04.058

31. Harmsen HJM, Elfferich P, Schut F, Welling GW (1999) A $16 \mathrm{~S}$ rRNA-targeted probe for detection of lactobacilli and enterococci in faecal samples by fluorescent in situ hybridization. Microb Ecol Health Dis 11:3-12. https://doi.org/10.1080/089106099435862

32. Langendijk PS, Schut F, Jansen GJ et al (1995) Quantitative fluorescence in situ hybridization of Bifidobacterium spp. with genusspecific 16S rRNA-targeted probes and its application in fecal samples. Appl Environ Microbiol 61:3069-3075

33. Zoetendal EG, von Wright A, Vilpponen-Salmela T et al (2002) Mucosa-associated bacteria in the human gastrointestinal tract are uniformly distributed along the colon and differ from the community recovered from feces. Appl Environ Microbiol 68:3401-3407

34. Vrhovsek U, Masuero D, Gasperotti M et al (2012) A versatile targeted metabolomics method for the rapid quantification of multiple classes of phenolics in fruits and beverages. J Agric Food Chem 60:8831-8840. https://doi.org/10.1021/jf2051569

35. Caporaso JG, Kuczynski J, Stombaugh J et al (2010) QIIME allows analysis of high-throughput community sequencing data. Nat Methods 7:335-336. https://doi.org/10.1038/nmeth.f.303

36. Caruso D, Visioli F, Patelli R et al (2001) Urinary excretion of olive oil phenols and their metabolites in humans. Metabolism 50:1426-1428. https://doi.org/10.1053/meta.2001.28073

37. Mateos R, Martínez-López S, Baeza Arévalo G et al (2016) Hydroxytyrosol in functional hydroxytyrosol-enriched biscuits is highly bioavailable and decreases oxidised low density lipoprotein levels in humans. Food Chem 205:248-256. https://doi. org/10.1016/j.foodchem.2016.03.011

38. González-Santiago M, Fonollá J, Lopez-Huertas E (2010) Human absorption of a supplement containing purified hydroxytyrosol, a natural antioxidant from olive oil, and evidence for its transient association with low-density lipoproteins. Pharmacol Res 61:364-370. https://doi.org/10.1016/j.phrs.2009.12.016

39. Pierno S, Tricarico D, Liantonio A et al (2014) An olive oilderived antioxidant mixture ameliorates the age-related decline of skeletal muscle function. Age Dordr Neth 36:73-88. https:// doi.org/10.1007/s11357-013-9544-9

40. Gerra G, Leonardi C, Cortese E et al (2007) Homovanillic acid (HVA) plasma levels inversely correlate with attention deficithyperactivity and childhood neglect measures in addicted patients. J Neural Transm Vienna Austria 1996 114:1637-1647. https://doi. org/10.1007/s00702-007-0793-6

41. Monagas M, Khan N, Andrés-Lacueva C et al (2009) Dihydroxylated phenolic acids derived from microbial metabolism reduce lipopolysaccharide-stimulated cytokine secretion by human peripheral blood mononuclear cells. Br J Nutr 102:201-206. https://doi.org/10.1017/S0007114508162110

42. Tuohy KM, Fava F, Viola R (2014) "The way to a man"s heart is through his gut microbiota'-dietary pro- and prebiotics for the management of cardiovascular risk. Proc Nutr Soc 73:172-185. https://doi.org/10.1017/S0029665113003911

43. Connolly ML, Tzounis X, Tuohy KM, Lovegrove JA (2016) Hypocholesterolemic and prebiotic effects of a whole-grain oatbased granola breakfast cereal in a cardio-metabolic "at risk" population. Front Microbiol 7:1675. https://doi.org/10.3389/ fmicb.2016.01675

44. Ryan PM, London LEE, Bjorndahl TC et al (2017) Microbiome and metabolome modifying effects of several cardiovascular disease interventions in apo-E(-/-) mice. Microbiome 5:30. https:// doi.org/10.1186/s40168-017-0246-x

45. Zamora-Ros R, Achaintre D, Rothwell JA et al (2016) Urinary excretions of 34 dietary polyphenols and their associations with lifestyle factors in the EPIC cohort study. Sci Rep 6:26905. https:// doi.org/10.1038/srep26905

46. Margalef M, Pons Z, Iglesias-Carres L et al (2016) Gender-related similarities and differences in the body distribution of grape seed flavanols in rats. Mol Nutr Food Res 60:760-772. https://doi. org/10.1002/mnfr.201500717 
47. Macready AL, George TW, Chong MF et al (2014) Flavonoidrich fruit and vegetables improve microvascular reactivity and inflammatory status in men at risk of cardiovascular diseaseFLAVURS: a randomized controlled trial. Am J Clin Nutr 99:479-489. https://doi.org/10.3945/ajen.113.074237

48. Catry E, Bindels LB, Tailleux A et al (2017) Targeting the gut microbiota with inulin-type fructans: preclinical demonstration of a novel approach in the management of endothelial dysfunction. Gut. https://doi.org/10.1136/gutjnl-2016-313316

49. Gorvitovskaia A, Holmes SP, Huse SM (2016) Interpreting prevotella and bacteroides as biomarkers of diet and lifestyle. Microbiome 4:15. https://doi.org/10.1186/s40168-016-0160-7
50. Nakayama J, Yamamoto A, Palermo-Conde LA et al (2017) Impact of westernized diet on gut microbiota in children on Leyte Island. Front Microbiol 8:197. https://doi.org/10.3389/ fmicb.2017.00197

51. Barbieri R, Coppo E, Marchese A et al (2017) Phytochemicals for human disease: an update on plant-derived compounds antibacterial activity. Microbiol Res 196:44-68. https://doi.org/10.1016/j. micres.2016.12.003 